\title{
Solutions to the 3D Transport Equation and 1D Diffusion Equation for Passive Tracers in the Atmospheric Boundary Layer and Their Applications
}

\author{
SHUZHAN REN \\ Environment and Climate Change Canada, Downsview, Ontario, Canada
}

(Manuscript received 31 July 2018, in final form 24 February 2019)

\begin{abstract}
A solution to the $3 \mathrm{D}$ transport equation for passive tracers in the atmospheric boundary layer $(\mathrm{ABL})$, formulated in terms of Green's function (GF), is derived to show the connection between the concentration and surface fluxes of passive tracers through GF. Analytical solutions to the 1D vertical diffusion equation are derived to reveal the nonlinear dependence of the concentration and flux on the diffusivity, time, and height, and are employed to examine the impact of the diffusivity on the diurnal variations of $\mathrm{CO}_{2}$ in the ABL. The properties of transport operator $H$ and their implications in inverse modeling are discussed. It is found that $H$ has a significant contribution to the rectifier effect in the diurnal variations of $\mathrm{CO}_{2}$. Since $\mathrm{H}$ is the integral of GF in time, the narrow distribution of GF in time justifies the reduction of the size of $H$ in inverse modeling. The exponential decay of GF with height suggests that the estimated surface fluxes in inverse modeling are more sensitive to the observations in the lower ABL. The solutions and first mean value theorem are employed to discuss the uncertainties associated with the concentrationmean surface flux equation used to link the concentrations and mean surface flux. Both analytical and numerical results show that the equation can introduce big errors, particularly when surface flux is sign indefinite. Numerical results show that the conclusions about the evolution properties of passive tracers based on the analytical solutions also hold in the cases with a more complicated diffusion coefficient and time-varying ABL height.
\end{abstract}

\section{Introduction}

Passive tracers are a special type of tracer gases in the atmosphere that do not couple to any meteorological field and have no feedback on their transport operator. In addition to the long-lived tracers such as $\mathrm{SF}_{6}$ and $\mathrm{C}_{2} \mathrm{~F}_{6}$, the deviation of $\mathrm{CO}_{2}, \mathrm{CH}_{4}$, and $\mathrm{CO}$ from the wellmixed global means may be considered approximately as passive tracers. While $\mathrm{CO}_{2}$ and $\mathrm{CH}_{4}$ are greenhouse gases that can affect atmospheric radiation and cause global warming in a long term, $\mathrm{CO}$ is a major air pollutant that can have a negative impact on human health. Because these passive tracers are emitted from Earth's surface, understanding the transport of passive tracers within the atmospheric boundary layer (ABL) is important not only for understanding the biosphere (Fung et al. 1983), global chemical budget and radiation balance (Prather 1996), but also for understanding the performance of inverse modeling in which surface fluxes to be estimated are connected to the passive

Corresponding author: Shuzhan Ren, shuzhan.ren@canada.ca tracer's concentrations through the transport (Gurney et al. 2003).

The temporal and spatial variations of the passive tracer's concentration in the ABL are determined by advection, surface fluxes, and the vertical turbulent mixing, among which the turbulent mixing is particularly important as it plays an important role in the transport of heat, momentum, and chemical species in the ABL. The turbulent mixing is associated with the atmospheric turbulence generated in an unstable atmospheric state during the exchange of heat and momentum between Earth's surface and the atmosphere. The spatial scales of turbulence vary from meters under the stable condition (defined by the surface virtual heat flux) to the depth of ABL under the unstable condition (Stull 1988). Turbulent mixing affects the passive tracer transport in two ways: inserting a friction force directly in the transport equation and changing the advection of tracers through affecting the momentum and temperature equations. In numerical weather prediction (NWP) models, the small scales of turbulent motions cannot be resolved and the subgrid-scale motions have to be parameterized 
based on some empirical assumptions (Louis et al. 1982; Williamson et al. 1987; Holtslag and Boville 1993). Uncertainties associated with the parameterization of turbulent mixing are a major source of errors in temperature and wind data assimilation analyses and forecasts, model predictions of passive tracer concentration and the surface flux estimates in inverse modeling (Gloor et al. 1999; Peylin et al. 2002; Lin and Gerbig 2005; Houweling et al. 2010; Kretschmer et al. 2012; Makar et al. 2014). Therefore, the performance of the transport models of passive tracers in the ABL is very sensitive to turbulent mixing.

While numerical models are widely employed to study the passive tracer transport in the ABL, the analytical solution to the transport equation, derived under idealized conditions, can be also useful in gaining a better understanding of the mechanism underlying the important features of the spatiotemporal variations of passive tracers as well as in better interpreting numerical results of the transport model. Furthermore, insight gained from the analytical solutions can help understand the role of the transport operator in estimating the surface sources and sinks of $\mathrm{CO}_{2}$, which is of vital importance for reliably predicting future $\mathrm{CO}_{2}$ levels and for $\mathrm{CO}_{2}$ control and management (Gurney et al. 2003, 2004; Baker et al. 2003; Butler et al. 2010).

In hydro-environment studies, analytical solutions to the transport equations have been widely applied to investigate the spatiotemporal variations of pollutants and other chemicals in the aquifers (Singh and Gupta 1984; Basha and El-Habel 1993; Zoppou and Knight 1999; Leij et al. 2000; Jaiswal et al. 2011; Chen and Liu 2011). In contrast, there are only few studies on the analytical solutions to transport equations for passive tracers in the ABL and their applications. Based on the analytical solution to the 3D transport model with zero surface flux and without the nonlocal effect, Holzer (1999) and Holzer and Hall (2000) investigated numerically the transport time scales, pathways, and mechanisms of passive tracers. Larson and Volkmer (2008) explored the cause of the rapid increase of the $\mathrm{CO}_{2}$ mixing ratio in the early morning and nearly constant low concentration during the daytime-the so-called rectifier effect. In spite of these studies, the quantitative description of the impact of the eddy diffusivity on the diurnal rectifier effect is still unavailable. In addition, observations show that the $\mathrm{CO}_{2}$ mixing ratio and flux have different vertical distributions (Davis et al. 2003) and the cause of the difference has not been investigated. Furthermore, the accuracy of the concentrationmean surface flux equation employed in inverse modeling has not been examined, and the properties of the transport operator-a function that links the mean surface flux and the concentration of passive-has not been investigated in previous work due to the lack of explicit form.

The purpose of this work is to derive the analytical solutions to the transport equation for passive tracers in the ABL and apply the solutions to analyze the mechanism underlying the variations of passive tracers as well as the uncertainties associated with the concentration-mean surface flux equation employed in inverse modeling. For such purpose, the solution to the $3 \mathrm{D}$ transport equation for passive tracers in the $\mathrm{ABL}$, formulated in terms of Green's function (GF), is derived first to show the dependence of the passive tracer's concentration on fluxes at boundaries, the diffusion coefficient, and GF. Then the analytical solutions to the 1D diffusion equation is derived with the time-varying and time- and specified-height-varying diffusion coefficients. Since the solutions show not only the dependence of concentration on diffusion coefficient and surface fluxes at vertical boundaries, but also the analytical form of GF at the lower vertical boundary, they can be employed to examine the impact of the vertical eddy diffusivity on the spatial and temporal variations of the concentration, flux, and transport operator of passive tracers, particularly on the rectifier effect observed in the tall tower observations and numerical simulations of $\mathrm{CO}_{2}$ (Bakwin et al. 1998; Davis et al. 2003; Denning et al. 1996a,b; Yi et al. 2000; Chen et al. 2004). Because the solutions to the 3D and 1D equations contain the explicit relationship between the concentration and surface fluxes of passive tracers, they can be employed to examine the accuracy the concentrationmean surface flux equation used in inverse modeling.

This paper is arranged as follows. The 3D transport equation and $1 \mathrm{D}$ diffusion equation are introduced in section 2 . In section 3 , the solution to the $3 \mathrm{D}$ equations with a general diffusion coefficient is derived first. Then the analytical solutions to the 1D diffusion model with time-varying and time- and specified-height-varying diffusion coefficients are derived. The analytical solution to the $1 \mathrm{D}$ diffusion equation with a time-varying diffusion coefficient is employed to discuss the evolution of the $\mathrm{CO}_{2}$ mixing ratio and $\mathrm{CO}_{2}$ flux in the $\mathrm{ABL}$ in section 4 . The properties of the transport operator, its effects on the Kalman gain, and the accuracy of the concentration-mean surface flux equation are discussed in section 5. The impacts of the time- and height-varying diffusion coefficient and time-varying ABL depth on the variations of the $\mathrm{CO}_{2}$ mixing ratio, flux, transport operator, and inverse modeling are examined, and the results are compared with the results based on the analytical solution in section 6 . Conclusions are given in the final section. 


\section{The 3D transport model and $1 D$ vertical diffusion model in the ABL}

The transport of passive tracers in the ABL can be described by the following equation:

$$
\frac{\partial \rho_{a} \psi}{\partial t}+\nabla \cdot\left(\mathbf{u} \rho_{a} \psi\right)=S,
$$

where $\rho_{a}$ is the air density, $\psi$ is the (mass) mixing ratio of passive tracers, $\mathbf{u}=(u, v, w)$ is the advection velocity, and $S$ is the source of passive tracers in the atmosphere. By using the mass conservation of $\rho_{a}$, one can obtain the following equation of the passive tracer mixing ratio

$$
\frac{\partial \psi}{\partial t}+\mathbf{u} \cdot \nabla \psi=\frac{S}{\rho_{a}} .
$$

In the ABL, the turbulence, characterized by disordered, eddying fluid motions over a wide range of length scales, has a significant impact on the transport of passive tracers. To separate the contributions of the resolved scale motions and subgrid turbulent motions to the transport of passive tracers, each variable in Eq. (2) is decomposed as

$$
(\psi, \mathbf{u})=(\bar{\psi}, \overline{\mathbf{u}})+\left(\psi^{\prime}, \mathbf{u}^{\prime}\right),
$$

where variables with an overbar are ensemble means and variables with a prime represent the subgrid components whose ensemble mean is zero. Substituting Eq. (3) into Eq. (2) yields

$$
\frac{\partial \psi}{\partial t}+\mathbf{u} \cdot \nabla \psi+\frac{\partial}{\partial z}\left(\overline{w^{\prime} \psi^{\prime}}\right)=\frac{S}{\rho_{a}} \equiv \Upsilon .
$$

Overbars are dropped from the ensemble-mean variables in Eq. (4). In deriving Eq. (4), we assume that the flow is nondivergent and the effects of the turbulence in the horizontal are much smaller based on the argument that the characteristic scales in the horizontal are much larger than those in the vertical.

Since $\overline{w^{\prime} \psi^{\prime}}$ is an unresolved subscale variable, it has to be parameterized. In the nonlocal scheme it can be parameterized as

$$
\overline{w^{\prime} \psi^{\prime}}=-K_{\psi}\left(\frac{\partial \psi}{\partial z}-\gamma_{\psi}\right),
$$

where $K_{\psi}$ is the parameterized diffusion coefficient $\left(\mathrm{m}^{2} \mathrm{~s}^{-1}\right)$ and $\gamma_{\psi}$ is a countergradient term describing the nonlocal effects from the vertical transport of eddies with the vertical scale comparable to the height of the ABL under unstable and convective conditions (Holtslag and Moeng 1991; Holtslag and Boville 1993).
Since the exchange of momentum and heat between the atmosphere and the surface of the earth varies with time and the vertical mixing changes with height, $K_{\psi}$ is a function of time and height in general. The term $\gamma_{\psi}=\gamma_{\psi}(x, y, t)$ depends on the ABL height and the surface fluxes of passive tracers.

An important task in the ABL parameterization is to determine $K_{\psi}$ and the ABL height. Different approaches have been proposed to determine $K_{\psi}$ including using the evolution of turbulent kinetic energy (TKE closure; Mellor and Yamada 1982) and other empirical relationships ( $K$-closure; e.g., Louis 1979). In the $K$-closure, $K_{\psi}$ depends on the vertical shear of wind and the Richardson number in the local scheme (Louis et al. 1982; Williamson et al. 1987), and on the ABL height $z_{T}$ and $z$ in the nonlocal scheme (Holtslag and Boville 1993). Like other parameterization schemes, which involve assumptions and approximations, the eddy approach is based on the scale separation assumption. When more than one scale is present, higherorder closure scheme has to be used.

Substituting Eq. (5) into Eq. (4), one obtains

$$
\frac{\partial \psi}{\partial t}+\mathbf{u} \cdot \nabla \psi-\frac{\partial}{\partial z}\left(K_{\psi} \frac{\partial \psi}{\partial z}\right)=\Upsilon-\gamma_{\psi} \frac{d K_{\psi}}{d z} .
$$

Equation (6) shows that within the domain, advection, vertical mixing, internal sources, and nonlocal effect contribute to the variation rate of passive tracers with time. In addition, it will be shown that the fluxes at horizontal and vertical boundaries also affect the concentration of passive tracers. When the spatial distribution of passive tracers is heterogeneous or when a frontal system penetrates into the ABL, advection can have a strong impact on the transport of passive tracers. The advection effects on the transport of $\mathrm{CO}_{2}$ have been discussed by (Yi et al. 2000; Hurwitz et al. 2004; Wang et al. 2005). Equation (6) also shows that only when $K_{\psi}$ varies with height can the nonlocal effect affect the time tendency of mixing ratio.

For the constant horizontal wind and zero vertical wind, namely, $\mathbf{u}=(U, V, 0)$, where $U$ and $V$ are constant, a new variable $\Psi$ satisfying $\psi(\mathbf{r}, t)=\Psi(z, t)$ $f_{1}(x-U t) f_{2}(y-V t)$, where $f_{i}$ is determined by initial conditions, can be introduced, from which Eq. (6) can be transformed into the following equation:

$$
\frac{\partial \Psi}{\partial t}=\frac{\partial}{\partial z}\left(K_{\psi} \frac{\partial \Psi}{\partial z}\right)+f_{1}^{-1} f_{2}^{-1}\left(\Upsilon-\gamma_{\psi} \frac{d K_{\psi}}{d z}\right) .
$$

Under the conditions that the horizontal advection is weak, landscape and its sources and sinks of passive tracers are relatively homogeneous, or the terrain is 
reasonably flat, Eq. (6) can be further simplified by ignoring the advection terms in Eq. (6). Under these conditions, Eq. (6) becomes the following 1D diffusion equation:

$$
\frac{\partial \psi}{\partial t}=\frac{\partial}{\partial z}\left(K_{\psi} \frac{\partial \psi}{\partial z}\right)+\Upsilon-\gamma_{\psi} \frac{d K_{\psi}}{d z} .
$$

\section{Solutions to the 3D transport model and 1D vertical diffusion model in the $A B L$}

\section{a. Solution to the $3 D$ transport equation in $A B L$}

We consider the following horizontal boundary conditions:

$$
\left.\psi \mathbf{u} \cdot \mathbf{n}\right|_{\Gamma_{v, i}}=J_{h i}, \quad i=1, \ldots, 4,
$$

where $\mathbf{n}$ is the unit vector normal to the boundary surface in vertical $\Gamma_{i}$, and $J_{h i}$ is a given function. The vertical boundary conditions at the boundary surface in horizontal $\Gamma_{h, j}$ are

$$
\begin{aligned}
\left.K_{\psi}\left[\frac{\partial \psi(z, t)}{\partial z}\right]\right|_{\Gamma_{h, j}} & =-J_{v, j}(x, y, t),\left.\quad w_{j}\right|_{\Gamma_{v, j}}=0, \\
j & =1,2
\end{aligned}
$$

where $w_{j}$ is the vertical velocity at $\Gamma_{v, j}$, and $J_{v, j}$ is the specified flux.

The equation of the 3D GF $G_{3 \mathrm{D}}\left(\mathbf{r}, t \mid \mathbf{r}^{\prime}, \tau\right)$ corresponding to Eq. (6) is (Greenberg 1971; Leij et al. 2000)

$$
-\frac{\partial G_{3 \mathrm{D}}}{\partial \tau}-\mathbf{u}_{0} \cdot \nabla G_{3 \mathrm{D}}-\frac{\partial}{\partial z^{\prime}}\left(K_{\psi} \frac{\partial G_{3 \mathrm{D}}}{\partial z^{\prime}}\right)=\delta(t-\tau) \delta\left(\mathbf{r}-\mathbf{r}^{\prime}\right),
$$

where $\mathbf{r}$ is the coordinate of the domain; $\mathbf{r}^{\prime}$ and $\tau$ are dummy variables corresponding to $\mathbf{r}$ and $t$, respectively; $\nabla_{0}$ is the operator in $\mathbf{r}^{\prime}$ coordinates; and $\mathbf{u}_{0}=d \mathbf{r}^{\prime} / d \tau$. Parameter $G_{3 \mathrm{D}}\left(\mathbf{r}, t \mid \mathbf{r}^{\prime}, \tau\right)$ satisfies the following horizontal and vertical boundary conditions:

$$
\begin{aligned}
\left.G_{3 \mathrm{D}}\right|_{\Gamma_{v, i}} & =G_{h i}, \quad i=1, \ldots, 4, \\
\left.K_{\psi}\left(\frac{\partial G_{3 \mathrm{D}}}{\partial z^{\prime}}\right)\right|_{\Gamma_{h, j}} & =0, \quad j=1,2,
\end{aligned}
$$

and zero initial condition

$$
G\left(\mathbf{r}, t \mid \mathbf{r}^{\prime}, \tau\right)=0, \quad t<\tau .
$$

Once $G_{3 \mathrm{D}}$ is obtained from Eqs. (11)-(14), the solution to Eq. (6) can be written as (appendix A)

$$
\begin{aligned}
\psi(\mathbf{r}, t)= & \int_{\Omega} G_{3 \mathrm{D}}\left(\mathbf{r}, t \mid \mathbf{r}^{\prime}, 0\right) \psi\left(\mathbf{r}^{\prime}, 0\right) d^{3} \mathbf{r}^{\prime}+\int_{0}^{t} \int_{\Omega} G_{3 \mathrm{D}}\left(\mathbf{r}, t \mid \mathbf{r}^{\prime}, \tau\right)\left(\Upsilon-\gamma_{\psi} \frac{d K_{\psi}}{d z^{\prime}}\right) d \tau d^{3} \mathbf{r}^{\prime} \\
& +\left.\sum_{i=1}^{4} \int_{\sigma_{i}} \int_{0}^{t} J_{h, i} G_{h i}\right|_{\Gamma_{v, i}} d \sigma_{v, i} d \tau-\left.\sum_{j=1}^{2} \int_{\sigma_{j}} \int_{0}^{t}(-1)^{(j+1)} J_{v, j} G_{3 \mathrm{D}}\right|_{\Gamma_{h, j}} d \sigma_{h, j} d \tau,
\end{aligned}
$$

where $\Omega$ is the volume of the domain and, $\sigma_{v, i}$ and $\sigma_{h, j}$ are the areas of the boundary surface in vertical and horizontal, respectively. The first term in Eq. (15) is the contribution of initial condition. The second term is the contributions of the internal source of passive tracers and the combined nonlocal effect and the vertical gradient of diffusion coefficient. This combined term acts as an external force, and its magnitude is proportional to the product of $\gamma_{\psi}$ and vertical gradient of $K_{\psi}$. Therefore, only when $K_{\psi}$ is a function of height can $\gamma_{\psi}$ affect tracer concentrations. The third and fourth terms are the contributions of the passive tracer flux at horizontal and vertical boundaries, respectively. Advection and vertical mixing affect the concentration through $G_{3 \mathrm{D}}$. It will be shown in section 5 that Eq. (15) can be further simplified when the domain is global in horizontal and semi-infinite in vertical.
Although it is difficult to obtain the analytical form of $G_{3 \mathrm{D}}$ in general, Eq. (15) is still very useful in understanding the connection between the concentrations of passive tracers in the atmosphere and the vertical fluxes in global inverse modeling (Gurney et al. 2003) (see further discussion in section 5). Equation (15) is more general than the solution of the $3 \mathrm{D}$ advectiondiffusion equation derived by Holzer and Hall (2000) with zero-flux boundary conditions and $\gamma_{\psi}=0$. Although solution of Holzer and Hall has been employed to identify transport time scales, pathways, and mechanisms of passive tracers in the atmosphere (Holzer 1999; Holzer and Hall 2000), it cannot be used in inverse modeling due to the lack of description of the relationship between concentration and surface flux.

It should be pointed out that the GF method can be applied to solve linear differential equations only. 
It cannot be extended to solve the transport equation of statistics of fluctuations, such as the RMS fluctuations and covariances, which are nonlinear partial differential equations.

In examining the impacts of vertical eddy diffusivity on the variations of concentration, flux and transport operator of passive tracers in the ABL, the analytical forms of GF are needed. In the following part, we will derive the analytical GFs in the 1D diffusion model with time-varying and height- and time-varying diffusion coefficients.

\section{b. Analytical solution to the $1 D$ diffusion equation with time-varying $K_{\psi}$}

Compared with NWP models, the 1D diffusion model for passive tracers has a much simpler form, which contains neither nonlinear terms nor equations describing chemical reactions. Furthermore, unlike NWP models, which often have coarse vertical solutions within the ABL, the 1D diffusion model can be used to compute the high-resolution vertical profiles of concentration and flux of passive tracers. For this reason, the 1D diffusion model has been widely employed as an important tool in the hydroenvironmental studies and other fields that involve the mixing process. In the studies, the analytical solutions to the $1 \mathrm{D}$ diffusion equation have been applied to investigate the transport of pollutants in the aquifers (Singh and Gupta 1984; Basha and El-Habel 1993; Zoppou and Knight 1999; Jaiswal et al. 2011; Chen and Liu 2011). The 1D diffusion model has also been used for the investigation of the impact of vertical eddy diffusivity on the variability $\mathrm{CO}_{2}$ concentration (Chen et al. 2004; Larson and Volkmer 2008) and $\mathrm{CO}_{2}$ budget within the ABL (Lee 1998; Pino et al. 2012), tracer-age distributions in the atmosphere (Holzer and Hall 2000), and surface energy budget studies. In these studies, analytical solutions with general surface flux boundary conditions and nonlocal effect are not derived.

In this part, the analytical solution to the $1 \mathrm{D}$ diffusion model with the time-varying $K_{\psi}$ is derived, and then employed to examine the impact of the vertical diffusivity on the variation of passive tracers. In deriving the analytical solution, the following upper and lower boundary conditions are used:

$$
\begin{gathered}
\left.K_{\psi}\left[\frac{\partial \psi(z, t)}{\partial z}\right]\right|_{z=z_{T}}=-\mathscr{J}_{t}(t), \\
\left.K_{\psi}\left[\frac{\partial \psi(z, t)}{\partial z}\right]\right|_{z=0}=-\mathscr{J}_{b}(t),
\end{gathered}
$$

where $\mathscr{J}_{b}$ represents surface flux describing the uptake and release of passive tracers from various sources/ sinks from land and oceans, $\mathscr{J}_{t}$ represents the flux at the entrainment at the top of ABL (Wyngaard and Brost 1984), and $z_{T}$ is the ABL height. In the convective $\mathrm{ABL}, z_{T}$ is time dependent, but it is assumed to be time independent in deriving analytical solution. The evolution of passive tracers under the time-dependent $z_{T}$ is investigated numerically in section 6 . When the domain is semi-infinity, $z_{T}=\infty$ and $\mathscr{J}_{t}=0$. By integrating Eq. (8) (with $\Upsilon=0$ and $\gamma_{\psi}=0$ ) and using the boundary conditions, Pino et al. (2012) examines the impact of different factors on the budget of $\mathrm{CO}_{2}$ within the ABL.

To assess the impact of the surface flux on the variations of concentration and flux of passive tracers, we assume that the background (reference) concentration is removed, and the following zero initial condition is used in this work:

$$
\psi(z>0, t=0)=0 .
$$

For a time-dependent diffusion coefficient, $K_{\psi}$ can be put as $K_{\psi}=T(t) D$, where $D\left(\mathrm{~m}^{2} \mathrm{~s}^{-1}\right)$ is a (positive) constant, and $T(t)$ is a dimensionless function of time representing the variation of eddy diffusivity due to diurnal variation of surface heat flux. If the internal source is absent (i.e., $\Upsilon=0$ ), Eq. (8) can be written as

$$
\frac{\partial \psi}{\partial t}=D T(t) \frac{\partial^{2} \psi}{\partial z^{2}} .
$$

By introducing a new variable $\hat{t}=\int^{t} T(\hat{\tau}) d \hat{\tau}$, Eq. (18) can be transformed into the following equation:

$$
\frac{\partial \psi}{\partial \hat{t}}=D\left(\frac{\partial^{2} \psi}{\partial z^{2}}\right)
$$

To keep the nature of the initial condition unchanged, it requires that $\hat{t}(t=0)=0$. To apply the Laplace transform, it also requires that $\hat{t} \rightarrow \infty$ when $t \rightarrow \infty$. To solve Eq. (19), we first apply the Laplace transform to obtain

$$
D \frac{\partial^{2} \hat{\psi}}{\partial z^{2}}-p \hat{\psi}=0
$$

where $\hat{\psi}(z, p)=\int_{0}^{\infty} \exp (-p \hat{t}) \psi(z, t) d \hat{t}$, and $p$ is a positive constant. Because Eq. (20) is a secondary-order ordinary differential equation with constant coefficients, it has the following analytical solution

$$
\hat{\psi}(z, p)=a_{1} \exp (q z)+a_{2} \exp (-q z),
$$

where $q=\sqrt{p / D}>0$ and $a_{i}(i=1,2)$ is a constant coefficient. 
By applying the Laplace transform to the boundary conditions in Eqs. (16a) and (16b), one has

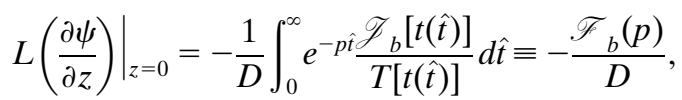

$$
\begin{aligned}
& \left.L\left(\frac{\partial \psi}{\partial z}\right)\right|_{z=z_{T}}=-\frac{1}{D} \int_{0}^{\infty} e^{-p \hat{t} \mathscr{J}_{t}[t(\hat{t})]} \frac{\mathscr{F}_{t}(p)}{T[t(\hat{t})]} d \hat{t} \equiv-
\end{aligned}
$$

Substituting Eq. (21) into Eqs. (22a) and (22b) yields

$$
\begin{aligned}
& a_{1}=\frac{\mathscr{F}_{b}(p) \exp \left(-q z_{T}\right)-\mathscr{F}_{t}(p)}{\sqrt{D q\left[\exp \left(q z_{T}\right)-\exp \left(-q z_{T}\right)\right]}}, \\
& a_{2}=\frac{\mathscr{F}_{b}(p) \exp \left(q z_{T}\right)-\mathscr{F}_{t}(p)}{\sqrt{D q}\left[\exp \left(q z_{T}\right)-\exp \left(-q z_{T}\right)\right]},
\end{aligned}
$$

from which $\hat{\psi}(z, p)$ can be expressed as

$$
\begin{aligned}
\hat{\psi}(z, p)= & \frac{\mathscr{F}_{b}(p)}{\sqrt{D p}} \frac{\cosh \left[\left(z_{T}-z\right) \sqrt{p} / \sqrt{D}\right]}{\sinh \left(z_{T} \sqrt{p} / \sqrt{D}\right)} \\
& -\frac{\mathscr{F}_{t}}{\sqrt{D p}} \frac{\cosh (z \sqrt{p} / \sqrt{D})}{\sinh \left(z_{T} \sqrt{p} / \sqrt{D}\right)} .
\end{aligned}
$$

By applying the inverse Laplace transform to Eq. (24) and using the following equation

$$
L^{-1}\left[\frac{1}{\sqrt{p}} \frac{\cosh (x \sqrt{p})}{\sinh (l \sqrt{p})}\right]=\frac{1}{l} \theta_{4}\left(\frac{x}{2 l} \mid \frac{i \pi \hat{t}}{l^{2}}\right), \quad-l \leq x \leq l,
$$

where $\theta_{4}(\mu \mid \eta)=(-i \eta)^{-1 / 2} \sum_{n=-\infty}^{n=\infty} \exp \left[-i \pi(\mu-0.5+n)^{2} / \eta\right]$ is the $\theta$ function (Bateman 1954) and $\eta \neq 0$, one obtains

$$
\begin{aligned}
\psi(z, t)= & \sum_{n=-\infty}^{n=\infty} \int_{0}^{t} \frac{1}{\sqrt{D \pi \mathscr{T}}} \exp \left[-\frac{\left(2 n z_{T}-z\right)^{2}}{4 D \mathscr{T}}\right] \mathscr{J}_{b}(\tau) d \tau \\
& -\sum_{n=-\infty}^{n=\infty} \int_{0}^{t} \frac{1}{\sqrt{D \pi \mathscr{T}}} \exp \left[\frac{-\left(z+(2 n-1) z_{T}\right)^{2}}{4 D \mathscr{T}}\right] \mathscr{J}_{t}(\tau) d \tau \\
\equiv & \int_{0}^{t} G_{1 \mathrm{D}, b}(z, t, \tau) \mathscr{J}_{b}(\tau) d \tau-\int_{0}^{t} G_{1 \mathrm{D}, t}(z, t, \tau) \mathscr{J}_{t}(\tau) d \tau
\end{aligned}
$$

where $\mathscr{T}=\int_{\tau}^{t} T\left(t^{\prime}\right) d t^{\prime}$ and $G_{1 \mathrm{D}, b}$ and $G_{1 \mathrm{D}, t}$ are the values of GF at the lower and upper boundary, respectively. In deriving Eq. (26), the convolution theorem for the Laplace transform is used. Note that when $\mathscr{T} \rightarrow 0, \psi$ is still bounded.

Equation (26) is a special case of the general solution (15). It shows that although the time derivative of the passive tracer concentration is proportional to $D T$, the concentration itself depends on the diffusivity and height in a highly nonlinear way. It also shows that the value of mixing ratio at time $t$ is affected not only by the surface flux at that time but also by the accumulative contribution of surface flux before $t$. This property has an important implication in inverse modeling for the surface flux estimate (see section 5).

Because $\psi$ decreases exponentially as $|n|$ increases, by taking $n=-1,0,1$ in the first integral and $n=0,1$ in the second integral, Eq. (26) can be approximated as

$$
\begin{aligned}
\psi(z, t) \approx & \int_{0}^{t} \frac{1}{\sqrt{D \pi \mathscr{T}}}\left\{\exp \left(-\frac{z^{2}}{4 D \mathscr{T}}\right)+\exp \left[-\frac{\left(2 z_{T}+z\right)^{2}}{4 D \mathscr{T}}\right]+\exp \left[-\frac{\left(2 z_{T}-z\right)^{2}}{4 D \mathscr{T}}\right]\right\} \mathscr{J}_{b}(\tau) d \tau \\
& -\int_{0}^{t} \frac{1}{\sqrt{D \pi \mathscr{T}}}\left\{\exp \left[-\frac{\left(z_{T}-z\right)^{2}}{4 D \mathscr{T}}\right]+\exp \left[-\frac{\left(z_{T}+z\right)^{2}}{4 D \mathscr{T}}\right]\right\} \mathscr{J}_{t}(\tau) d \tau .
\end{aligned}
$$

In Eq. (27), the contributions associated with the surface and entrainment fluxes are described by the first and second integrals, respectively. The first integral contains three terms. The first term represents the combined impact of surface flux and diffusivity on the vertical distribution of the concentration of passive tracers. In this term, the diffusivity leads to the decay of the mixing ratio with height. The second term represents the effect of the upper boundary on the bottom-up flux. The variation of this term with height is similar to that of the first 
term but with a smaller magnitude. The third term, also associated with the upper boundary, represents the impact of the reflected top-down flux originating from the surface flux on the vertical distribution of the concentration of passive tracers. The impact of this term is much smaller than the impact of the first term in the lower part of the ABL. Its variation with height is completely different from that of the first and second terms.

Two terms are contained in the second integral. The first term represents the effects of diffusivity and entrainment flux on the concentration of passive tracers. It increases exponentially with height. The second term describes the effect of the reflected top-down flux by the lower boundary on the variation of passive tracer concentration. It decreases with increasing height with a similar magnitude to that of the first term in the lower part of $A B L$ and a much smaller magnitude in the upper part.

In a semi-infinite domain $\left(z_{T} \rightarrow \infty\right)$ and Eq. (27) can be further simplified as

$$
\begin{aligned}
\psi(z, t) & =\int_{0}^{t} \frac{1}{\sqrt{D \pi \mathscr{T}(t, \tau)}} \exp \left[-\frac{z^{2}}{4 D \mathscr{T}(t, \tau)}\right] \mathscr{J}_{b}(\tau) d \tau \\
& \equiv \int_{0}^{t} \tilde{G}_{1 \mathrm{D}, b}(z, t \mid 0, \tau) \mathscr{J}_{b}(\tau) d \tau
\end{aligned}
$$

Because $\tilde{G}_{1 \mathrm{D}, b}(z, t \mid 0, \tau)$ in Eq. (28) is a highly nonlinear function of the diffusion coefficient, height, and time, the diffusion coefficient has different impact on the GF at different height, and the diurnal variation of $\psi$ at a given height is not symmetric in time even if the surface flux $\mathscr{J}_{b}$ is. In addition, because $\tilde{G}_{1 \mathrm{D}, b}$ is a function of $t, \psi$ does not always increases with time. Equation (28) is similar to the solution of the Stokes problem with similar boundary conditions (Batchelor 2000).

\section{c. Analytical solution with a time-varying and slowly height-varying $K_{\psi}$}

To examine the impact of the spatial variation of $K_{\psi}$ on the distributions of passive tracers in the ABL, it is assumed that $K_{\psi}=T(t) D(z)$, where

$$
D(z)=D_{0}\left[1+\varepsilon \frac{z}{z_{T}}\left(1-\frac{z}{z_{T}}\right)\right]
$$

and $\varepsilon$ is a small positive constant. Thus, $D$ is a slowly varying function of $z$ which has a similar form to that of the diffusion coefficient proposed by O'Brien (1970), and it has the maximum value $D_{\max }=D_{0}(1+\varepsilon / 4)$ at $z=z_{T} / 2$.

By using a new variable $\hat{t}=\int^{t} T(\tau) d \tau$, Eq. (8) can be put as

$$
\frac{\partial \psi}{\partial \hat{t}}=\frac{\partial}{\partial z}\left[D(z)\left(\frac{\partial \psi}{\partial z}-\gamma_{\psi}\right)\right]
$$

With some mathematical transformations (appendix B), Eq. (29) can be transformed into the following form

$$
\frac{\partial \phi}{\partial \hat{t}}=D(z) \frac{\partial^{2} \phi}{\partial z^{2}}+\Gamma \phi-\gamma_{\psi} \sqrt{D} \frac{d D}{d z},
$$

where $\phi=\sqrt{D} \psi$ and $\Gamma=D_{z}^{2} /(4 D)-D_{z z} / 2$. Because $\varepsilon$ is a small parameter,

$$
\Gamma=\frac{\varepsilon D_{0}}{z_{T}^{2}}+\mathscr{O}\left(\varepsilon^{2}\right) \approx \frac{\varepsilon D_{0}}{z_{T}^{2}}
$$

can be regarded as a constant. Thus, by introducing a new variable $\Phi=\phi \exp (-\Gamma \hat{t})$, Eq. (30) can be transformed into the following form

$$
\frac{\partial \Phi}{\partial \hat{t}}=D(z) \frac{\partial^{2} \Phi}{\partial z^{2}}-\gamma_{\psi} \sqrt{D} \frac{d D}{d z} \exp (-\Gamma \hat{t}) .
$$

Equation (32) is different from Eq. (19) in that $D$ is a function of height and $\gamma_{\psi}$ affects mixing ratio within the domain. Because $D(z)$ is a slowly varying function of height, the WKBJ method (Gill 1982), along with the Laplace transform, can be employed to derive the approximate solution under the boundary conditions [Eqs. (16a) and (16b)]. The complete solution can be obtained after doing lengthy derivations (appendix C) as

$$
\begin{aligned}
\psi(z, t)= & \lambda \sum_{n=-\infty}^{n=\infty} \int_{0}^{t} \frac{\mathscr{J}_{b}}{\sqrt{D \pi \mathscr{T}}} \exp \left\{-\frac{\left[2 n K_{0}\left(z_{T}\right)-K_{0}(z)\right]^{2}}{4 D_{0} \mathscr{T}}\right\} \exp (\Gamma \mathscr{T}) d \tau \\
& -\lambda \sum_{n=-\infty}^{n=\infty} \int_{0}^{t} \frac{\mathscr{J}_{t}}{\sqrt{D \pi \mathscr{T}}} \exp \left\{\frac{-\left[K_{0}(z)+(2 n-1) K_{0}\left(z_{T}\right)\right]^{2}}{4 D_{0} \mathscr{T}}\right\} \exp (\Gamma \mathscr{T}) d \tau+\varepsilon \int_{0}^{t} \gamma_{\psi} \mathscr{\mathscr { T }} d \tau \\
\equiv & \int_{0}^{t} \mathscr{J}_{b}(\tau) \mathscr{G}_{b}(z, t, \tau) d \tau-\int_{0}^{t} \mathscr{J}_{t}(\tau) \mathscr{G}_{t}(z, t, \tau) d \tau+\varepsilon \int_{0}^{t} \gamma_{\psi}(\tau) \mathscr{G}(z, t, \tau) d \tau,
\end{aligned}
$$


where $\mathscr{G}$ is defined in Eq. (C10) and

$\lambda=\left[\frac{D(z)}{D_{0}}\right]^{1 / 4}, \quad K_{0}(z)=\int^{z}\left[1+\varepsilon \frac{z^{\prime}}{z_{T}}\left(1-\frac{z^{\prime}}{z_{T}}\right)\right]^{-1 / 2} d z^{\prime}$.

Equation (33) is the special case of the more general solution in (15). The first and second terms in Eq. (33) are the contributions of the fluxes at lower and upper boundaries to the mixing ratio, respectively, which are similar to the first and second terms in Eq. (26). The third term describes the impact of the nonlocal effect. Equation (33) shows that not only $D$ but also its vertical variation can have an impact on the mixing ratio. The first derivative of $D$, contained in $\mathscr{E}_{1}(z)$, which is defined in Eq. (D6), leads to the presence of $\psi_{\gamma}$ in Eq. (33). The results show that the value of first derivative at bottom (contained in $\mathscr{E}_{0}$, which is defined in appendix D) enhances passive tracer concentrations, and the value within the domain reduces concentrations in the lower half of the ABL in which $\mathscr{E}_{1}(z)$ is negative, but enhances concentrations in the upper half of the ABL in which $\mathscr{E}_{1}(z)$ is positive. The second derivative, contained in $\Gamma$, which is defined in Eq. (31), leads to the exponential increase of mixing ratio in the whole domain. Because the passive tracer flux is proportional to the gradient of mixing ratio, the variation of $D$ has similar impact on flux.

For a deep ABL (very large $z_{T}$ ), the contribution of the second term to the mixing ratio in the lower $A B L$ is very small and the first term is also very small except for $n=0$. If the nonlocal effect is weak Eq. (33) becomes

$$
\psi \approx-\lambda \int_{0}^{t} \frac{\mathscr{J}_{b}}{\sqrt{D(z) \pi \mathscr{T}}} \exp \left[-\frac{K_{0}^{2}(z)}{4 \mathscr{T} D_{0}}\right] \exp (\Gamma \mathscr{T}) d \tau
$$

Comparison of Eqs. (34) and (28) suggests that the two solutions have similar forms when the ABL height is large. The differences between these two are associated with the spatial variation of $D$. Since $D_{0}$ is dominant in $D$, the modulation of $\psi$ by the spatial variation of $D$ should be small.

\section{Variation of $\mathrm{CO}_{2}$}

Among all the terms in Eq. (27), the first term is the dominant in the lower part of the ABL. Thus, Eq. (28) is a good approximation of the solution to the 1D diffusion equation, and it will be used in this section to analyze the rectifier effect observed in the time series of $\mathrm{CO}_{2}$, and the impact of vertical mixing on the variation of $\mathrm{CO}_{2}$ mixing ratio, and the features of $\mathrm{CO}_{2}$ flux.

\section{a. The rectifier effect in $\mathrm{CO}_{2}$ variation}

The rectifier effect is the feature seen in the diurnal variations of the $\mathrm{CO}_{2}$ mixing ratio in the lower part of the ABL over the vegetation regions. The time series of the $\mathrm{CO}_{2}$ mixing ratio shows a rapid increase in the early hours of the morning [0000-0600 local time (LT)] and persistent low values in a long period during the day. This asymmetric feature is in contrast to the quasisymmetric variation of the surface vegetation flux in time, and is associated directly with the variation of eddy diffusivity in the ABL. Larson and Volkmer (2008) discussed this effect qualitatively. In the following, Eq. (28) is used to explain this effect.

In the early morning, the surface flux of $\mathrm{CO}_{2}$ from vegetation respiration is positive and the diffusion is weak. Therefore, the accumulation of $\mathrm{CO}_{2}$ in the lower part of $\mathrm{ABL}$ causes a strong vertical gradient of $\mathrm{CO}_{2}$ concentration. These features can be described by Eq. (28). Because $\mathscr{J}_{b}$ and $D$ are positive constants in the early morning before $t_{c}$ when the surface flux changes its sign, Eq. (28) can be written as

$$
\psi\left(z, t \leq t_{c}\right) \approx \mathscr{J}_{b 0} \int_{0}^{t} \tilde{G}_{1 \mathrm{D}, b}(z, \tau) d \tau .
$$

Because $\tilde{G}_{1 \mathrm{D}, b}(z, \tau)$ is positive, integral (35) increases as time $t$ increases. Thus, $\psi$ increases with time at a given height and $D$, and the increase is more evident in the lower ABL due to the decrease of the $\tilde{G}_{1 \mathrm{D}, b}$ with height. Close to the surface, $\tilde{G}_{1 \mathrm{D}, b}(z, \tau) \approx 1 / \sqrt{D \tau}$, therefore $\psi\left(z, t \leq t_{c}\right) \approx 2 \mathscr{J}_{b 0} \sqrt{t} / \sqrt{D \pi}$.

At sunrise, the increase of the $\mathrm{CO}_{2}$ mixing ratio stops when diffusion starts to increase rapidly due to the strong exchange of heat and momentum between the ground and the atmosphere. $\mathrm{CO}_{2}$ is lifted upward by strong mixing into the upper part of $\mathrm{ABL}$ and thus significantly reduces the $\mathrm{CO}_{2}$ concentration near the ground. The reduction is further accelerated by the change of the surface flux from positive to negative (due to photosynthesis of vegetation), and then becomes small until the diffusivity becomes weak in the late afternoon. During this period, strong mixing makes the vertical distribution of the $\mathrm{CO}_{2}$ mixing ratio homogeneous. To apply Eq. (28) to describe these significant changes, we rewrite Eq. (28) as

$$
\begin{aligned}
\psi\left(z, t>t_{c}\right)= & \mathscr{J}_{b 0} \int_{0}^{t_{c}} \tilde{G}_{1 \mathrm{D}, b}(z, t, \tau) d \tau \\
& +\int_{t_{c}}^{t} \tilde{G}(z, t, \tau)_{1 \mathrm{D}, b}(\tau) \mathscr{J}_{b 1} d \tau,
\end{aligned}
$$

where $\mathscr{J}_{b 1}$ is the surface flux after $t_{c}$, which is negative. Close to the surface the value of the $\tilde{G}_{1 \mathrm{D}, b}$ is determined 
approximately by $1 / \sqrt{D \mathscr{T}}$, therefore $\psi\left(z, t>t_{c}\right)$ can be approximated as

$$
\begin{aligned}
\psi\left(z, t>t_{c}\right) \approx & \frac{2 \mathscr{J}_{b 0}}{\sqrt{D \pi}}\left[\sqrt{t+Z_{0}\left(t_{c}, t\right)}-\sqrt{t-t_{c}+Z_{0}\left(t_{c}, t\right)}\right] \\
& -\int_{t_{c}}^{t} \tilde{G}_{1 \mathrm{D}, b}(z, t, \tau)\left|\mathscr{J}_{b 1}(\tau)\right| d \tau
\end{aligned}
$$

where $Z_{0}\left(t_{c}, t\right)=\int_{t_{c}}^{t} T\left(\tau^{\prime}\right) d \tau^{\prime}$. In deriving Eq. (37), $T(t)=1$ between $0 \mathrm{~h}$ and $t_{c}$ and $\mathscr{T}\left(\tau, t>t_{c}\right)=t-\tau+$ $Z_{0}\left(t_{c}, t\right)$ are used.

Because $\psi\left(z, t_{c}\right) \approx 2 \mathscr{J}_{b 0} \sqrt{t_{c}} / \sqrt{D \pi}$ and $\sqrt{t_{c}}>$ $\sqrt{t+Z_{0}\left(t_{c}, t\right)}-\sqrt{t-t_{c}+Z_{0}\left(t_{c}, t\right)}$,

$$
\psi\left(z, t_{c}\right)>\psi\left(z, t>t_{c}\right),
$$

suggesting that the rapid increase of diffusion at sunrise, along with the change of $\mathscr{J}_{b}$ to negative, would cause a big drop in $\psi$ after $t_{c}$. Although $\tilde{G}_{1 \mathrm{D}, b}$ increases in the upper ABL as diffusion increases, $\psi$ can still decrease after $t_{c}$ in the upper ABL due to the negative $\mathscr{J}_{b 1}$. The decrease of $\psi$ then continues until late afternoon but becomes slow due to the slow variation of the diffusion coefficient. During this period, the strong vertical mixing leads to slow the decay of $\tilde{G}_{1 \mathrm{D}, b}$ with height as the decay rate is proportional to $D^{3 / 2}$. Consequently, the vertical distribution of the $\psi$ becomes more homogeneous in vertical. In the late afternoon, $\psi$ gradually increases as diffusion becomes weak and $\mathscr{J}_{1 b}$ changes from negative to positive.

\section{b. Impact of diffusivity on the diurnal variation of the biospheric $\mathrm{CO}_{2}$ mixing ratio}

Although the variation rate of concentration is proportional to diffusion coefficient, concentration itself depends on the diffusion coefficient in a highly nonlinear way as shown by Eq. (28). This nonlinear relationship is further examined numerically in this part based on Eq. (28) and an observed biogenic $\mathrm{CO}_{2}$ flux. In the numerical calculation, $T$ is assumed to be the following piecewise function:

$$
T(t)=\left\{\begin{array}{cc}
1, & 6 \geq t \geq 0 \mathrm{~h} \\
1+\beta \sin \left(\frac{\pi}{2} \frac{t}{12}\right), & 24>t>6 \mathrm{~h},
\end{array}\right.
$$

where $t$ is the local standard time (LST) and $\beta$ is a positive constant. Thus, $D$ represents the value of $K_{\psi}$ during nighttime and in early morning, and $D(1+\beta)$ measures the maximum diffusion coefficient during the daytime. The value of $K_{\psi}$ has a jump at $6 \mathrm{~h}$, reflecting the sudden increase of vertical mixing. It starts to increase from $6 \mathrm{~h}$, reaches a maximum at $12 \mathrm{~h}$, and then decays to $D$ at $24 \mathrm{~h}$, corresponding to the diurnal variation of surface heat flux. Using Eq. (39) $\mathscr{T}(t, \tau)$ can be obtained as

$$
\mathscr{T}(t, \tau)=\left\{\begin{array}{cc}
t-\tau, & 6 \geq t>0 \mathrm{~h} \\
t-\tau+\frac{24 \beta}{\pi} Z(t, \tau), & 24 \geq t>6 \mathrm{~h},
\end{array}\right.
$$

where $Z(t, \tau)=\cos (6 \pi / 24)-\cos (\pi t / 24)>0$ when $\tau<6 \mathrm{~h}$ and $Z(t, \tau)=\cos (\pi \tau / 24)-\cos (\pi t / 24)>0$ when $\tau>6 \mathrm{~h}$.

Because we are only interested in the diurnal variation of $\mathrm{CO}_{2}$ mixing ratio, surface flux of $\mathrm{CO}_{2}$ is assumed to have zero daily mean. It is constructed based on the eddy covariance measurements of the net ecosystem-atmosphere exchange (NEE) $\left(\mathrm{mg} \mathrm{m}^{-2} \mathrm{~s}^{-1}\right.$ or $22.72 \mu \mathrm{mol} \mathrm{m}^{-2} \mathrm{~s}^{-1}$ ) near the surface $(30 \mathrm{~m})$ from the tall towers in Chequamegon National Forest in northern Wisconsin (Yi et al. 2000; Gerritsen 2012) as

$$
\mathscr{J}_{b}(t)=\left\{\begin{array}{clrl}
0.25, & 6 \geq t>0 \mathrm{~h} \\
0.25-0.35(t-6 \mathrm{~h}), & & t_{n} \geq t>6 \mathrm{~h} \\
-0.35, & 24-t_{n} \geq t>t_{n} \mathrm{~h} \\
-0.35+0.35\left[t-\left(24-t_{n}\right) \mathrm{h}\right], & & 18 \geq t>\left(24-t_{n}\right) \mathrm{h} \\
0.25, & 24 \geq t>18 \mathrm{~h},
\end{array}\right.
$$

where $t_{n}=54 / 7$ is the time when the surface flux first reaches -0.35 . In Eq. (41), the positive constant flux between 18 and $6 \mathrm{~h}$ and negative constant between 7 and $17 \mathrm{~h}$ reflect the positive and negative fluxes associated with nighttime respiration and daytime photosynthesis of $\mathrm{CO}_{2}$, respectively. In the following numerical calculation, $\mathscr{J}_{b} / \rho_{0}$ (where $\rho_{0}$ is the air density near the surface) is used and the unit of $\psi$ is converted from mass ratio to parts per million by volume.

To test the impact of the diffusion coefficient on the evolution of the $\mathrm{CO}_{2}$ mixing ratio, $D=1,2,10$, and 100 and $\beta=10$ are used to compute the diurnal variations of $\mathrm{CO}_{2}$ at different heights based on Eq. (28). The results at 30,120 , and $390 \mathrm{~m}$ are presented in Fig. 1. It can be seen from the figure that strong diffusion tends to dampen 

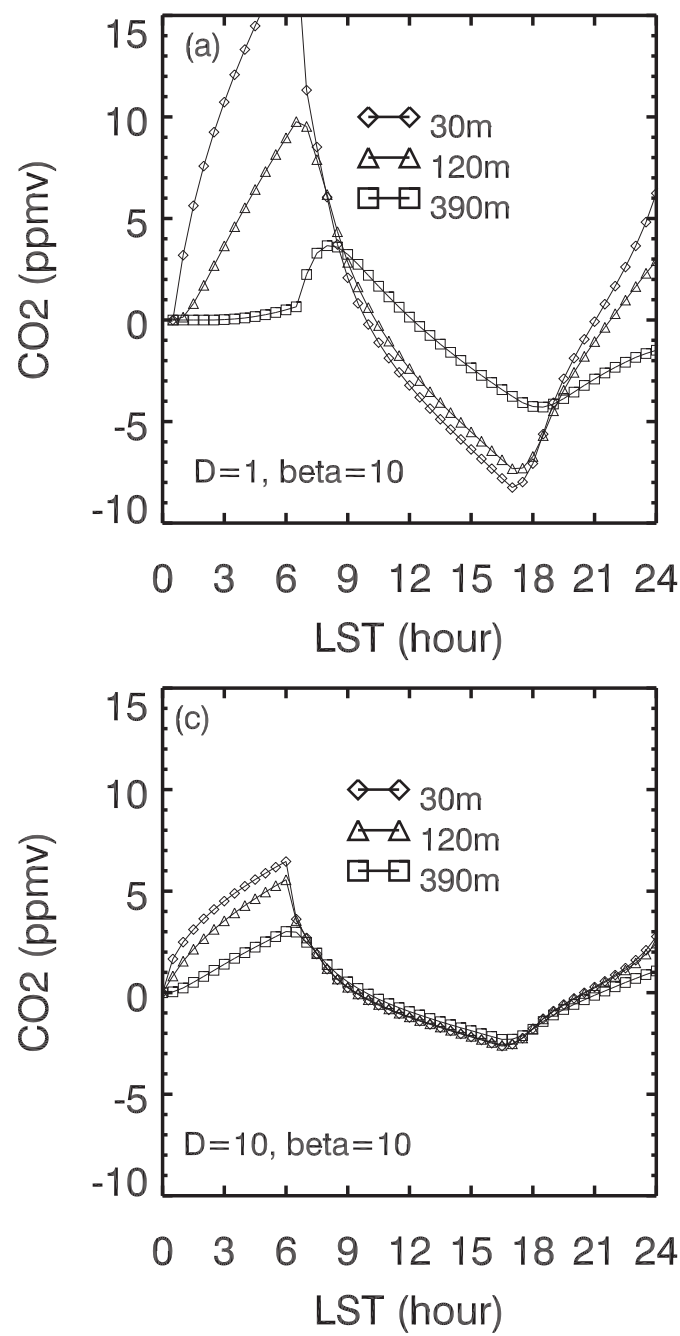
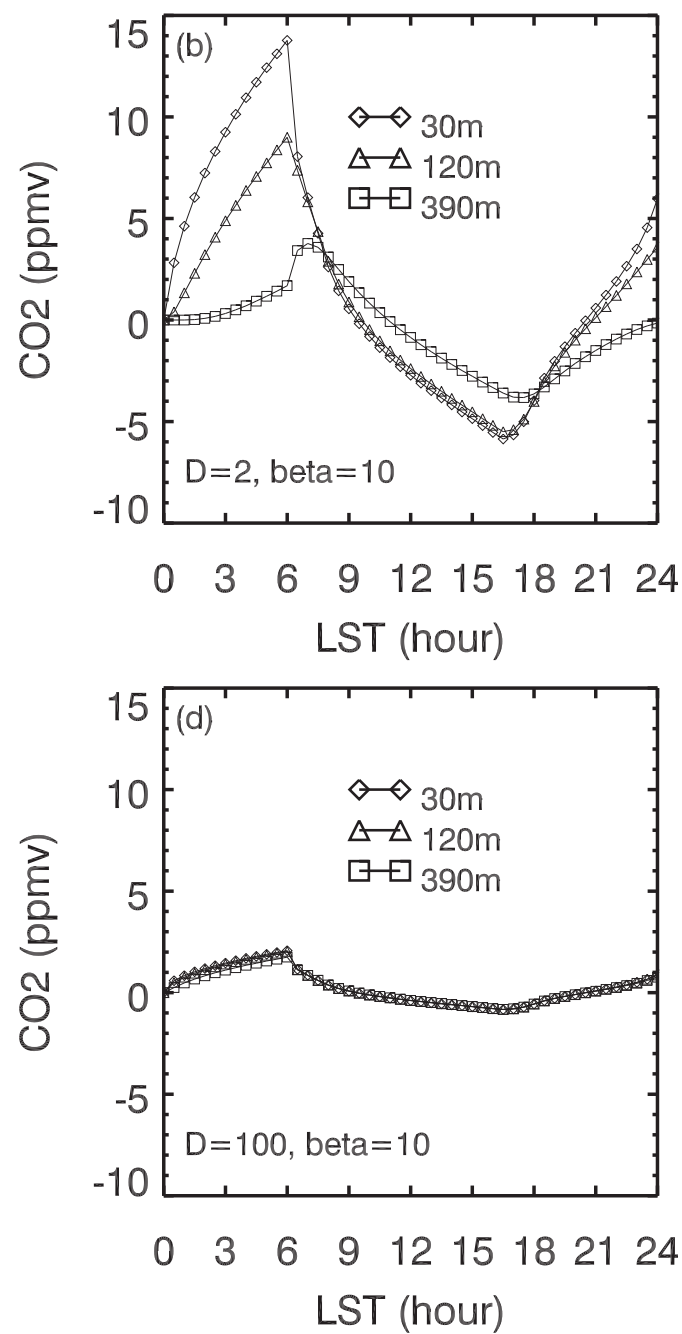

FIG. 1. Diurnal variation of the $\mathrm{CO}_{2}$ mixing ratio (ppmv) computed from Eq. (28) at different heights with $\beta=10$ and (a) $D=1$, (b) $D=2$, (c) $D=10$, and (d) $D=100$.

mixing ratio at every level. When $D$ (the diffusion coefficient in the early morning) is small $(D=1,2)$ there is a clear decay of the $\mathrm{CO}_{2}$ mixing ratio with height in the early morning. Corresponding to the jump of $K_{\psi}$ at around $t=6 \mathrm{~h}$, the mixing ratios at 30 and $100 \mathrm{~m}$ drop very quickly.

The large differences in daytime mixing ratio at different height shown in Fig. 1 do not match the observations. This suggests that $\beta=10$ is not strong enough to slow down the decay rate during the daytime. This mismatch can be greatly reduced when $\beta=40$ is used in the calculation as shown in Fig. 2. With this value, the mixing ratio during the daytime is more uniform in vertical for $D=1-2$. For a large $D(D=10,100)$ the variation of $\mathrm{CO}_{2}$ is strongly dampened in the whole day and the rectifier effect is very weak. These results suggest that in order to describe the reasonable rectifier effect, the magnitude of the diffusion coefficient should be 1 or 2 during the nighttime and early morning and 40 during the daytime.

Comparison between Figs. $2 \mathrm{a}$ and $2 \mathrm{~b}$ and tall tower observations shown in Fig. 1 in Yi et al. (2000) suggests that with $D=1$ or 2 and $\beta=40$, the analytical results capture the features of the evolution patterns of the observed $\mathrm{CO}_{2}$ mixing ratio at different levels. The tall tower observations show that from 0100 to $0600 \mathrm{LT}$, the $\mathrm{CO}_{2}$ mixing ratio increases by 15 ppmv from 370 to 385 ppmv at $30 \mathrm{~m}$, and by 5 ppmv from 360 to $365 \mathrm{ppmv}$ at $122 \mathrm{~m}$. These two increased values agree with those shown in Figs. 2a and $2 \mathrm{~b}$ at 30 and $120 \mathrm{~m}$. At high level (around $400 \mathrm{~m}$ ), both observations and numerical results show that $\mathrm{CO}_{2}$ mixing ratio is almost a constant from 0100 to $0600 \mathrm{LT}$, and has about $2.5 \mathrm{ppmv}$ increases from 0600 to $0900 \mathrm{LT}$. After $0600 \mathrm{LT}, \mathrm{CO}_{2}$ mixing ratios at 30 and $120 \mathrm{~m}$ go through two distinct stages, and the evolution patterns can be identified in both observations 

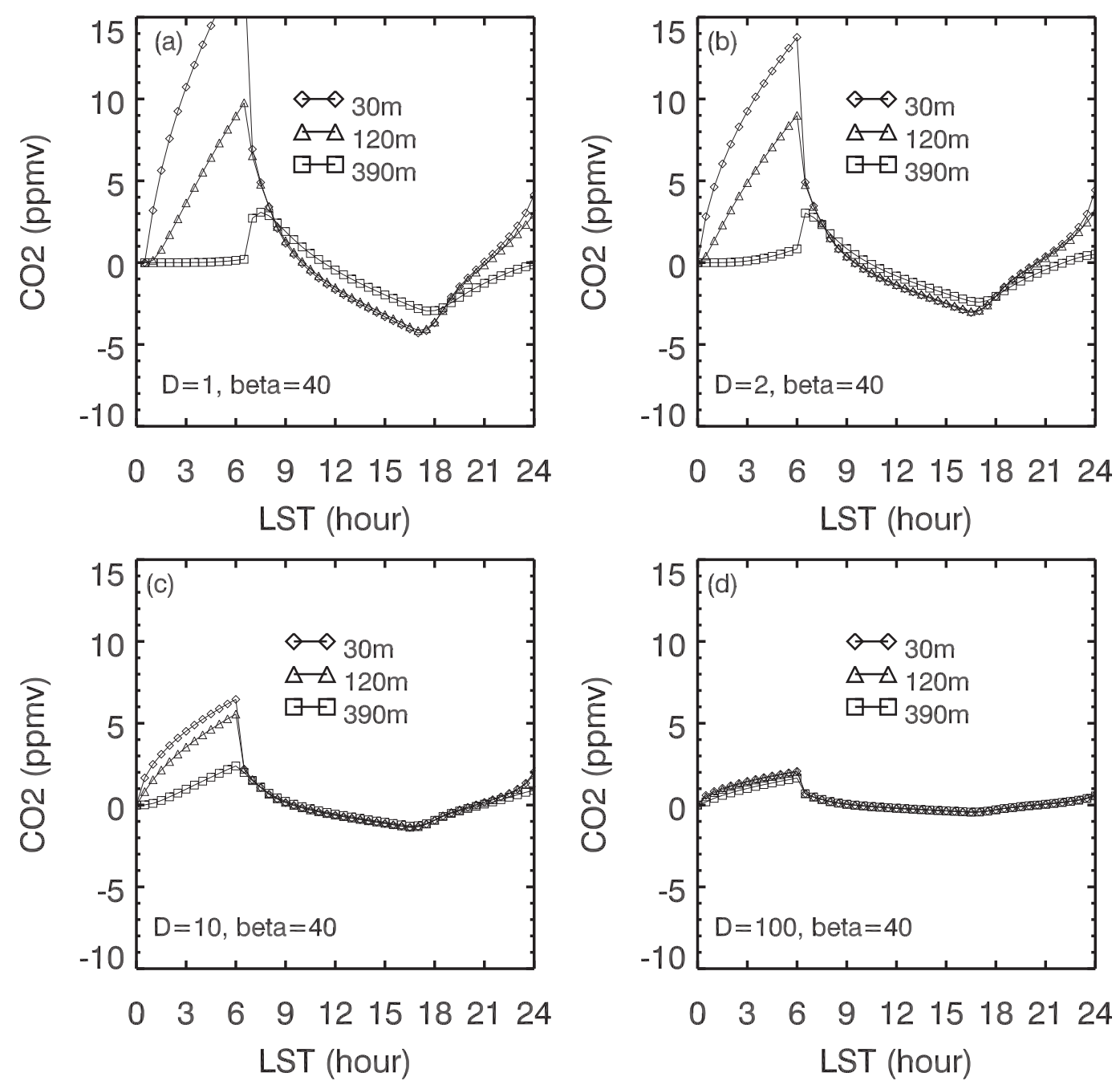

FIG. 2. As in Fig. 1, but with $\beta=40$.

and numerical results. In the first stage, from 0600 to $0900 \mathrm{LT}, \mathrm{CO}_{2}$ mixing ratios drop about $20 \mathrm{ppmv}$ at $30 \mathrm{~m}$ and $10 \mathrm{ppmv}$ at $120 \mathrm{~m}$ in both observations and numerical results. In the second stage, the vertical distribution of mixing ratio becomes homogeneous due to the rectifier effect, and the homogeneous mixing ratio continues to decay by about 5 ppmv until the sunset time (1800 LT). The similarities suggest that with the proper diffusion coefficient and surface flux, the analytical solution in (28) can be employed to gain the physical insight into the underline mechanism of the $\mathrm{CO}_{2}$ mixing ratio in the ABL. In addition, evolution pattern of $\mathrm{CO}_{2}$ in Figs. 2a and $2 \mathrm{~b}$ is also very close to the numerical results shown in Fig. 10 in (Chen et al. 2004) based on the ABL model.

\section{c. Diurnal variation of $\mathrm{CO}_{2}$ flux}

Observations show that while the $\mathrm{CO}_{2}$ mixing ratio decays with height monotonically during the daytime, the magnitude of turbulent flux of $\mathrm{CO}_{2}$ can increase with height (Yi et al. 2000). To understand the different vertical distributions, we first derive the $\mathrm{CO}_{2}$ flux from Eq. (28) as

$$
\begin{aligned}
\overline{w^{\prime} \psi^{\prime}}(z, t) & =-K_{\psi} \frac{\partial \psi}{\partial z}=\int_{0}^{t} \frac{z T(t)}{2 \mathscr{T}} G_{1 \mathrm{D}, b}(z, t, \tau) \mathscr{J}_{b}(\tau) d \tau \\
& \equiv \int_{0}^{t} G_{F}(z, t, \tau) \mathscr{J}_{b}(\tau) d \tau .
\end{aligned}
$$

Because $G_{F}$ in Eq. (42) contains the product of $z$ and $\exp \left(-z^{2} / 4 D \mathscr{T}\right)$, it should increase with height when $z<\sqrt{2 D \mathscr{T}}$. Thus, $G_{F}$ is quite different from $G_{1 \mathrm{D}, b}$ which decreases monotonically with increasing height. This property can be seen more clearly in Fig. 3a which shows that when $D=0.2$ the magnitude of $\mathrm{CO}_{2}$ flux at $z=$ $390 \mathrm{~m}$ is smaller than that at $z=30 \mathrm{~m}$ and $z=120 \mathrm{~m}$ before $13 \mathrm{~h}$, and it becomes larger than that at $z=30 \mathrm{~m}$ 

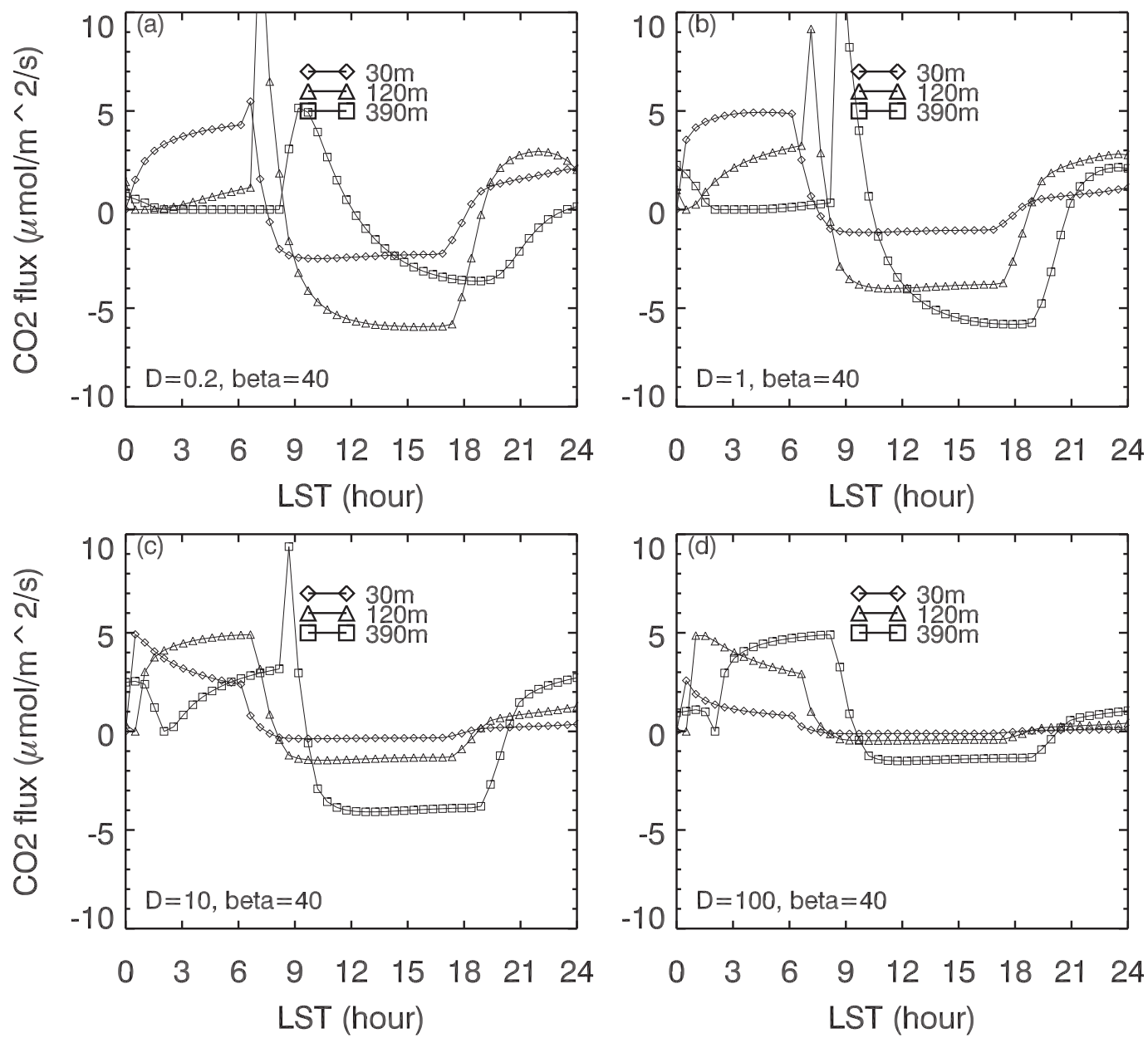

FIG. 3. Diurnal variation of the $\mathrm{CO}_{2}$ flux computed from Eq. (42) at different height with $\beta=40$ and (a) $D=0.2$, (b) $D=1$, (c) $D=10$, and (d) $D=100$.

after $13 \mathrm{~h}$. When $D=2$ (Fig. $3 \mathrm{~b}$ ), it becomes the largest after $12 \mathrm{~h}$. Figure 3 also shows that the diffusivity has less impact on the vertical distribution of the $\mathrm{CO}_{2}$ flux during the daytime. For large diffusion coefficients $(D=10,100)$, the vertical distribution of the flux is still much less uniform than that of the mixing ratio (Figs. 3c,d).

Comparison between Fig. $3 \mathrm{~b}$ and observation of turbulent flux of $\mathrm{CO}_{2}$ (Fig. 2b in Yi et al. 2000) shows that while flux at $z=390 \mathrm{~m}$ agrees with observed flux during the daytime, there are two differences. The first difference is that the magnitude of flux at $z=30 \mathrm{~m}$ is the smallest during the daytime in the 1D model, but the observed magnitude is the largest only before $12 \mathrm{~h}$. The difference may be due to the fact that the parameterization scheme used in the flux calculation is derived based on the flux equation with some terms in the equation being discarded (Holtslag and Moeng 1991), whereas it is the directly observed variable in (Yi et al. 2000) which should include the contributions of all the terms in the flux equation. In addition, since $z=30 \mathrm{~m}$ is within the surface boundary layer, the constant diffusion coefficient may be inadequate to describe thermodynamical processes that affect the $\mathrm{CO}_{2}$ flux.

Another difference is the flux in the morning. Figure $3 b$ shows that corresponding to the change of mixing ratio, fluxes at $z=120 \mathrm{~m}$ and $390 \mathrm{~m}$ have a big jump in the morning around 6 and $9 \mathrm{~h}$, respectively. However, these jumps are absent in the observations. Again this difference may be due to the parameterization scheme used in the 1D model.

\section{Applications of analytical solutions in inverse modeling}

In inverse modeling, observations of the $\mathrm{CO}_{2}$ concentration in the atmosphere are used as a constraint in estimating the time-mean surface $\mathrm{CO}_{2}$ flux such as the annual mean (Gurney et al. 2003, 2004; Baker et al. 2003), monthly mean (Bruhwiler et al. 2005) and weekly mean (Peters et al. 2005) surface fluxes. The mean 
surface $\mathrm{CO}_{2}$ flux can be estimated by minimizing a cost function as

$$
\mathbf{J}_{b, M}=\mathbf{J}_{b, p}+\mathbf{w}\left(\mathbf{H}^{-1} \mathbf{c}-\mathbf{J}_{b, p}\right),
$$

where $\mathbf{H}$ is the combination of a transport operator [see definition in Eq. (48)] and interpolation operator which is different from the interpolation operator in optimal interpolation (OI) with order-one magnitude (Daley 1993); $\mathbf{c}$ is a vector of the observations minus the initial $\mathrm{CO}_{2}$ concentrations; $\mathbf{J}_{b, p}$ and $\mathbf{J}_{b, M}$ are the prior timemean surface flux and the time-mean flux to be estimated (in vector form), respectively;

$$
\mathbf{w}=\frac{\mathbf{Q H}^{\mathrm{T}} \mathbf{H}}{\mathbf{H Q H}^{\mathrm{T}}+\mathbf{R}}
$$

is the Kalman gain; $\mathbf{Q}$ represents the uncertainties of $J_{p}$; and $\mathbf{R}$ represents the combination of observation errors, model errors, and representation errors. The representation errors contain the interpolation errors and errors associated with the concentration-surface flux equation (see discussion in section $5 b$ ).

It can be seen from Eq. (43) that the Kalman gain is a measure of the sensitivity of the analysis increment $\left(\mathbf{J}_{b, M}-\mathbf{J}_{b, p}\right)$ to the observation innovation $\left(\mathbf{H}^{-1} \mathbf{c}-\mathbf{J}_{b, p}\right)$. Its magnitude describes the strength of the constraint of observations on the estimated surface flux. As the Kalman gain increases as $\mathbf{H}$ increases, observations at different height would have different constraints on the estimated surface flux. Due to the important role of the transport operator in inverse modeling, the analytical solutions will be used in the following part to discuss the temporal and spatial variations of the transport operator under the influence of diffusivity, the implication of the variations for the Kalman gain, and the accuracy of the concentration-surface flux equation.

\section{a. Impact of diffusivity on the transport operator}

\section{1) TRANSPORT OPERATOR}

To discuss the $3 \mathrm{D}$ transport operator, the domain is assumed to be semi-infinite in vertical, and thus contribution from the upper vertical boundary is zero. After introducing a new variable

$$
\begin{aligned}
\Delta \psi \equiv & \psi(\mathbf{r}, t)-\int_{\Omega} G_{3 \mathrm{D}}\left(\mathbf{r}, t \mid \mathbf{r}^{\prime}, 0\right) \psi\left(\mathbf{r}^{\prime}, 0\right) d^{3} \mathbf{r}^{\prime} \\
& -\int_{0}^{t} \int_{\Omega} G_{3 \mathrm{D}}\left(\mathbf{r}, t \mid \mathbf{r}^{\prime}, \tau\right) \mathbf{r} d \tau d^{3} \mathbf{r}^{\prime} \\
& -\left.\sum_{i=1}^{4} \int_{\sigma_{i}} \int_{0}^{t} J_{h, i} G_{h i}\right|_{\Gamma_{v, i}} d \sigma_{v, i} d \tau
\end{aligned}
$$

to define the tracer concentration after deducting the background values including initial value contribution (the first integration on the right), contributions from the internal sources (the second integral), and contributions from four horizontal fluxes (the third integral), the analytical solution to the 3D passive tracer transport equation can be written as

$$
\Delta \psi=-\int_{0}^{t} \int_{\Omega} G_{3 \mathrm{D}} \gamma_{\psi} \frac{d K_{\psi}}{d z^{\prime}} d \tau d^{3} \mathbf{r}^{\prime}+\int_{\sigma_{b}} \int_{0}^{t} J_{b} G_{3 \mathrm{D}, b} d \sigma_{b} d \tau .
$$

Because $\gamma_{\psi}$ is proportional to $J_{b}$, namely, $\gamma_{\psi}=\gamma^{\prime} J_{b}$, Eq. (46) can be put as

$$
\Delta \psi=\int_{0}^{t} \int_{\sigma_{b}}\left(G_{3 \mathrm{D}, b}+\gamma_{0,3 \mathrm{D}}\right) J_{b} d \sigma_{b} d \tau \equiv \int_{\sigma_{b}} \int_{0}^{t} \tilde{G}_{3 \mathrm{D}} J_{b} d \sigma_{b} d \tau,
$$

where $\gamma_{0,3 \mathrm{D}}=-\int_{0}^{\infty} \gamma^{\prime} G_{3 \mathrm{D}}\left(d K_{\psi} / d z^{\prime}\right) d z^{\prime}$ is the contribution of nonlocal effect.

In inverse modeling, instead of computing $\tilde{G}_{3 \mathrm{D}}$, the concept of transport operator is introduced to connect the concentration of passive tracer in the atmosphere and the time averaged surface flux. The transport operator at a given point (or a specified area) is constructed by integrating a transport model with a unit surface flux at that point (or a prescribed area) and zero surface flux elsewhere (Gurney et al. 2003; Bruhwiler et al. 2005). It is used in inverse modeling to sample the value of the model output at the observation locations and times, and is affected by advection, diffusion and nonlocal effects. From Eq. (47) the 3D transport operator (or basis function) $H_{3 \mathrm{D}}$ can be defined as

$$
H_{3 \mathrm{D}}=\int_{0}^{t} \tilde{G}_{3 \mathrm{D}} d \tau
$$

Thus, the 3D transport operator is determined not only by the GF at the lower boundary but also by the $3 \mathrm{D}$ GF and the vertical derivative of diffusion coefficient within the domain if the nonlocal effect is included.

In the $1 \mathrm{D}$ case (with zero initial condition and internal source), Eq. (47) becomes

$$
\Delta \psi(z, t)=\int_{0}^{t}\left(\tilde{G}_{1 \mathrm{D}, b}+\gamma_{0,1 \mathrm{D}}\right) \mathscr{J}_{b} d \tau,
$$

where $\gamma_{0,1 \mathrm{D}}=-\int_{0}^{\infty} \gamma^{\prime} G_{1 \mathrm{D}}\left(d K_{\psi} / d z^{\prime}\right) d z^{\prime}$ is a general form of the third term on the right-hand side of Eq. (33). Thus, the $1 \mathrm{D}$ transport operator can be defined as 


$$
H_{1 \mathrm{D}}=\int_{0}^{t}\left(\tilde{G}_{1 \mathrm{D}, b}+\gamma_{0,1 \mathrm{D}}\right) d \tau
$$

\section{2) IMPACT OF DIFFUSIVITY}

To examine the impact of diffusivity on the transport operator quantitatively, the following $H_{1 \mathrm{D}}$ with the time-varying diffusion coefficient is used:

$$
H_{1 \mathrm{D}}(t, z)=\int_{0}^{t} \tilde{G}_{1 \mathrm{D}, b}(t, \tau, z) d \tau
$$

where $\tilde{G}_{1 \mathrm{D}, b}$ is defined in Eq. (28). Because $1 / \sqrt{D \mathscr{T}}$ and $\exp \left(-z^{2} / 4 D \mathscr{T}\right)$ in $\tilde{G}_{1 \mathrm{D}, b}$ vary differently with $D \mathscr{T}$, it can be proved that for a given $t, \tilde{G}_{1 \mathrm{D}, b}$ has a maximum

$$
\tilde{G}_{1 \mathrm{D}, b, \max }(z)=\sqrt{2 / e \pi} / z \approx 0.48 / z,
$$

when $\tau=\tau_{c}$, where $\tau_{c}$ is determined by $2 D \mathscr{T}\left(t, \tau_{c}\right)=z^{2}$. Equation (52) shows that $\tilde{G}_{1 \mathrm{D}, b, \max }$ is inversely proportional to height. Although the diffusion coefficient does not have impact on $\tilde{G}_{1 \mathrm{D}, b, \text { max }}$, it can change the time when the maximum is reached.

The variation of $\tilde{G}_{1 \mathrm{D}, b}$ with $\tau$ at $t=24 \mathrm{~h}$ at different height is computed and presented in Fig. 4 . The figure shows that the time series of $\tilde{G}_{1 \mathrm{D}, b}$ narrowly distributes around $\tau_{c}$ which is close to $t=24 \mathrm{~h}$, and the value of $\tilde{G}_{1 \mathrm{D}, b}$ near $\tau_{c}$ is inversely proportional to $z$, agreeing with Eq. (52). The narrow distribution of $\tilde{G}_{1 \mathrm{D}, b}$ around $\tilde{G}_{1 \mathrm{D}, b, \text { max }}$ suggests that only the surface flux near $\tau_{c}$ has a dominant contribution to $H_{1 \mathrm{D}}$ and the contribution few hours before $\tau_{c}$ can be neglected. This property justifies the reduction of the size of matrix of $H_{1 \mathrm{D}}$ in inverse modeling (Bruhwiler et al. 2005).

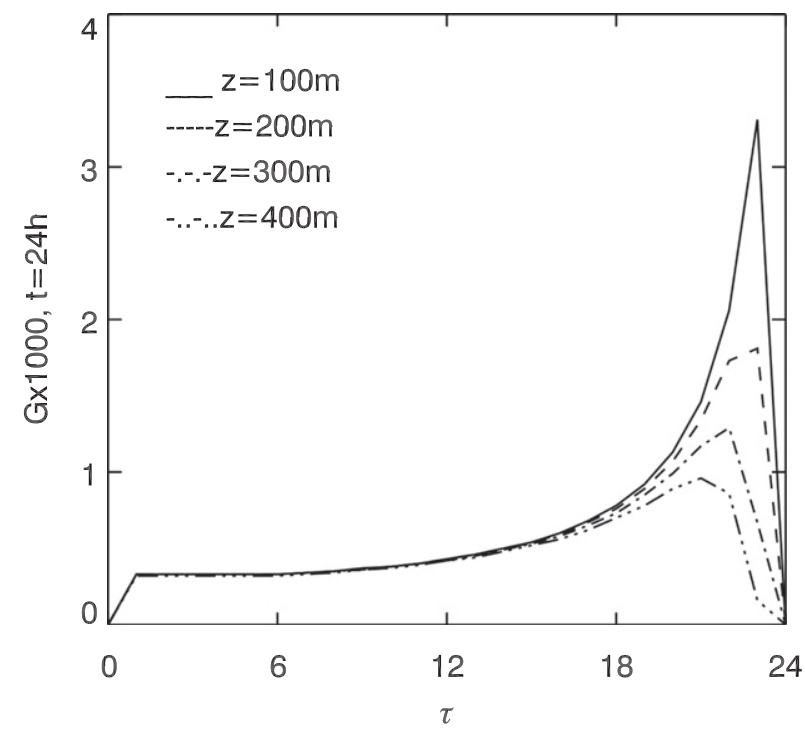

FIG. 4. Variation of $\tilde{G}_{1 \mathrm{D}, b}(\times 1000)$ with time $\tau$ at $t=24 \mathrm{~h}$ at different heights.

The variations of $H_{1 \mathrm{D}}$ with time $t$ at different height are shown in Fig. 5a $(D=1, \beta=40)$ and Fig. 5 c $(D=10$, $\beta=40)$. Comparison of Figs. 5a and 2a shows that the spatial and temporal distributions of $H_{1 \mathrm{D}}$ in the lower $\mathrm{ABL}$ are quite similar to those of the $\mathrm{CO}_{2}$ mixing ratio with a rapid increase in the early morning before $6 \mathrm{~h}$, a big drop after $6 \mathrm{~h}$, and small variation in vertical during the daytime. The reason for the rapid increases of $H$ before $6 \mathrm{~h}$ is due to the accumulation of $G_{1 \mathrm{D}, b}$ over time, similar to the reason for the increase of mixing ration. To understand the drop after $6 \mathrm{~h}$, we put Eq. (51) near the surface as

$$
\begin{aligned}
H_{1 \mathrm{D}}\left(z, t>t_{c}\right) & =\int_{0}^{t_{c}} \tilde{G}_{1 \mathrm{D}, b}(t, \tau, z) d \tau+\int_{t_{c}}^{t} \tilde{G}_{1 \mathrm{D}, b}(t, \tau, z) d \tau \\
& \approx \frac{2}{\sqrt{D \pi}}\left[\sqrt{t+\beta_{1} Z\left(t_{c}, t\right)}-\sqrt{t+\beta_{1} Z\left(t_{c}, t\right)-t_{c}}\right] \\
& +\frac{2}{\sqrt{D \pi}} \int_{t_{c}}^{t} \frac{d \tau}{\sqrt{t-\tau+\beta_{1} Z(t, \tau)}},
\end{aligned}
$$

where $\beta_{1}=24 \beta / \pi, t_{c}=6$, and $Z$ is defined in Eq. (40). Because $\sqrt{t_{c}}>\sqrt{t+\beta_{1} Z\left(t_{c}, t\right)}-\sqrt{t+\beta_{1} Z\left(t_{c}, t\right)-t_{c}}$ and $H_{1 \mathrm{D}}\left(z, t>t_{c}\right)$ decreases as $t+\beta_{1} Z\left(t_{c}, t\right)$ increases, for a strong vertical mixing, there exists a $t$ beyond which $H_{1 \mathrm{D}}$ has a drop in the lower ABL, namely,

$$
H_{1 \mathrm{D}}\left(z, t_{c}\right)-H_{1 \mathrm{D}}\left(z, t>t_{c}\right)>0 .
$$

The similarity between Eqs. (38) and (54) suggests that the rectifier effect observed in the lower ABL is mainly due to the mixing process described by the operator. However, the similarity appears only in the lower ABL. Figure 5a show that in the upper ABL, $H_{1 \mathrm{D}}(h=570$ and $840 \mathrm{~m}), H_{1 \mathrm{D}}$ increases monotonically with time. The different evolution patterns of $H_{1 \mathrm{D}}$ and mixing ratio are due to the different surface flux used in $H_{1 \mathrm{D}}$ and $\mathrm{CO}_{2}$ mixing ratio computations.

According to Eq. (44), for given $\mathbf{Q}$ and $\mathbf{R}$, the Kalman gain decreases as $\mathbf{H}$ decreases. Figures $5 \mathrm{~b}$ and $5 \mathrm{~d}$ show that $H_{1 \mathrm{D}}$ decreases with increasing height in general. 

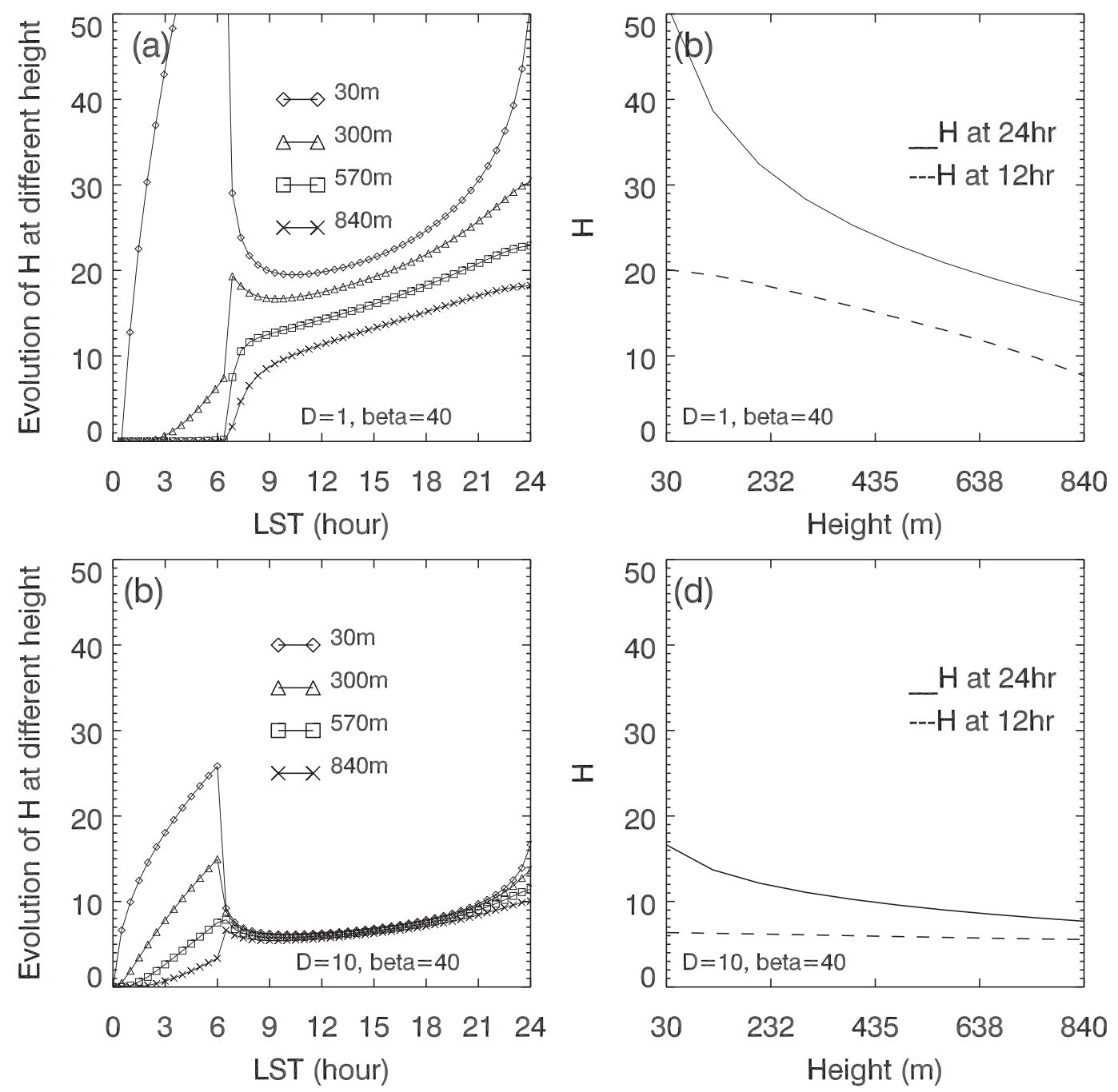

FIG. 5. Variation of $H_{1 \mathrm{D}}$ computed from Eq. (50) with time at different heights with $\beta=40$ and (a) $D=1$ and (c) $D=10$, and the variation of $H_{1 \mathrm{D}}$ at $t=12$ (dashed line) and $24 \mathrm{~h}$ (solid line) with height with (b) $D=1$ and (d) $D=10$.

It tends to amplify $\mathbf{w}$ at lower levels and reduce $\mathbf{w}$ at higher levels. All these properties suggest that the estimated mean fluxes are more sensitive to observations at lower levels.

\section{b. Discussion on the concentration-mean surface flux equation in inverse modeling}

Although Eq. (43) looks similar to the equation used in the conventional data assimilation scheme (Daley 1993), they are still very different in that while the variable to be estimated and the variable used as the constraint are the same variable or are connected through a given function in the data assimilation scheme, the two variables in inverse modeling are different and are not connected through an explicit function. In addition, analytical solutions show that there is no one-to-one correspondence in time and space between the $\mathrm{CO}_{2}$ concentration in the atmosphere and surface fluxes as the concentration at a given time is the accumulated contributions of fluxes before that time from multiple locations. Therefore, assumptions have to be made in constructing the concentration-mean surface equation to connect the concentration in the atmosphere and surface fluxes. In this part, analytical solutions are used to discuss the assumption and associated uncertainties.

Two approaches can be employed to link the averaged surface flux and the concentration of passive tracers depending how observations are used. In the first approach, the time-varying transport operator is used to sample the model results at the observation sites and at the observation times. In this approach the 

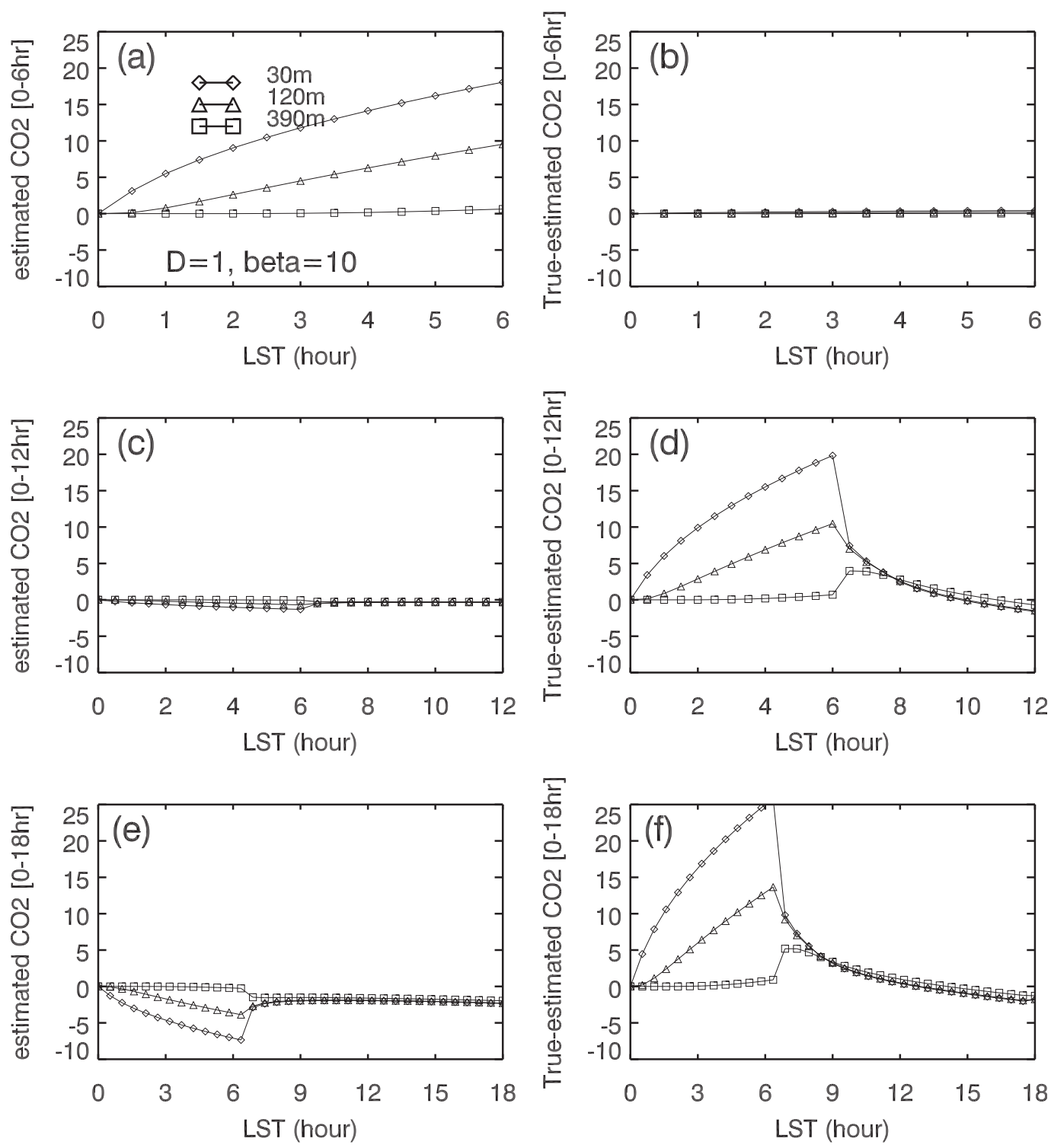

FIG. 6. The estimated $\mathrm{CO}_{2}$ mixing ratio at 30,120 , and $390 \mathrm{~m}$ based on $H_{1 \mathrm{D}} \overline{\mathscr{J}}_{b}$, where $\overline{\mathscr{J}}_{b}$ is the averaged $\mathscr{J}_{b}$ over (a) $6 \mathrm{~h}$ (starting from $0 \mathrm{~h}$ ), (c) $12 \mathrm{~h}$, and (e) $18 \mathrm{~h}$. (b),(d),(f) Corresponding differences between the true and the estimated mixing ratios.

concentration is computed from the transport operator and the averaged surface flux $J_{b}$ through the following concentration-flux equation

$$
\Delta \psi^{\prime}(z, t)=\int_{\sigma_{b}} H_{3 \mathrm{D}}(z, t) \bar{J}_{b}\left(\sigma_{b}, t\right) d \sigma_{b},
$$

where $\sigma_{b}$ is the horizontal area. Obviously Eq. (55) is different from Eq. (47) unless $J_{b}$ is a constant. To see this more clearly, we first consider the case in which $J_{b}$ is sign indefinite and $\tilde{G}_{3 \mathrm{D}, b}$ is positive. In this case we can apply the first mean value theorem for integration to Eq. (47) to obtain

$$
\Delta \psi=\int_{\sigma_{b}} J_{b, m} \int_{0}^{t} \tilde{G}_{3 \mathrm{D}}(z, \tau) d \tau d \sigma_{b}=\int_{\sigma_{b}} H_{3 \mathrm{D}} J_{b, m} d \sigma_{b},
$$

where $J_{b, m}$ is the value of $J_{b}$ at a particular time between 0 and $t$ depending on the distribution of $\tilde{G}_{3 \mathrm{D}, b}$. Due to this dependence, $\tilde{G}_{3 \mathrm{D}, b}$ and is not the averaged surface flux, and Eq. (56) is different from Eq. (55).

Now consider the case in which $J_{b}$ is sign definite. In this case, Eq. (47) can be put into the following form 

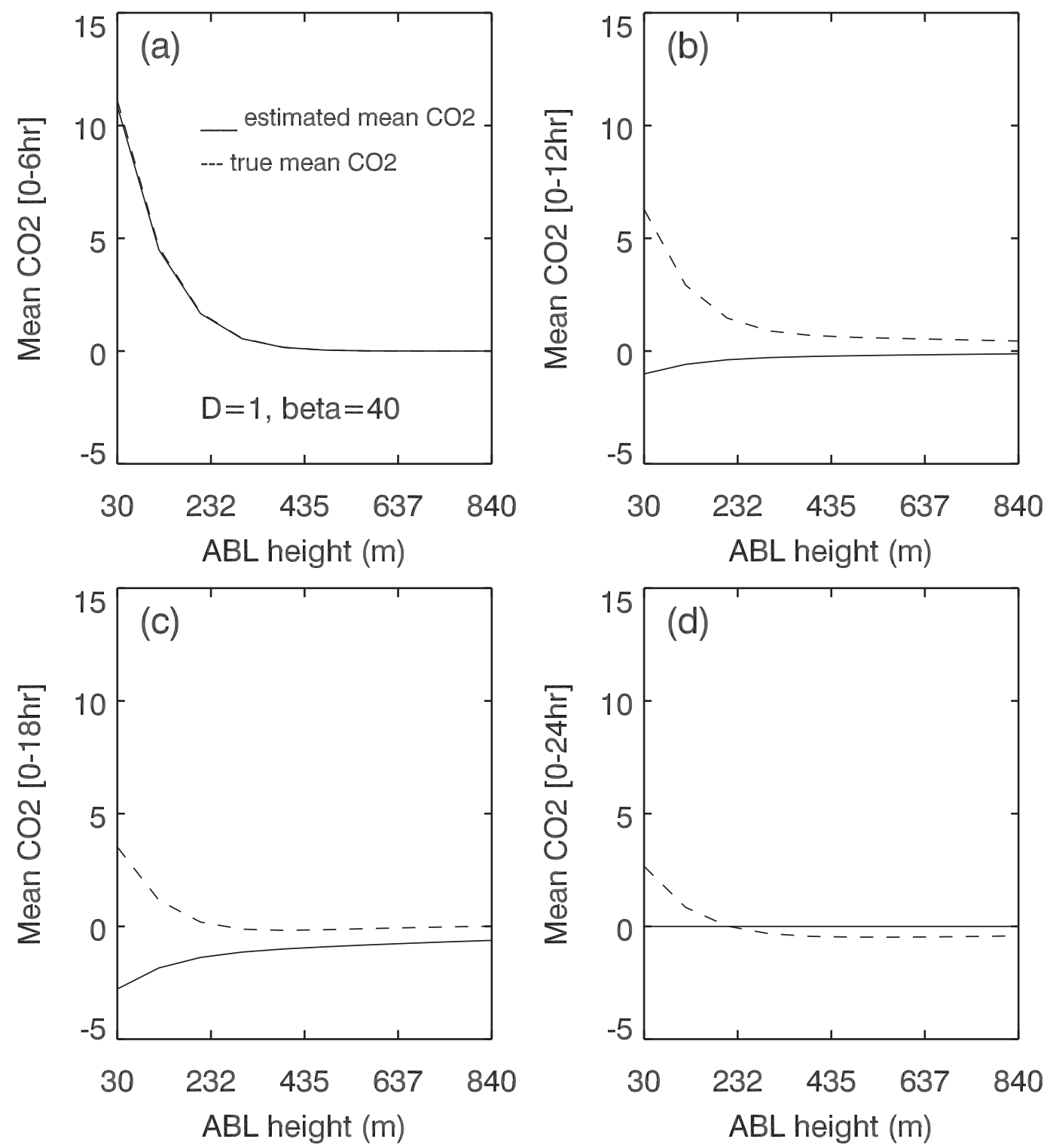

FIG. 7. Variations of the estimated time-mean $\mathrm{CO}_{2}$ mixing ratio computed from $\bar{H}_{1 \mathrm{D}} \overline{\mathscr{J}}_{b}$ (dashed lines) and the time-mean $\mathrm{CO}_{2}$ mixing ratio computed from Eq. (18) with $\gamma_{\psi}=0$ (solid lines) with height over (a) 6, (b) 12, (c) 18 , and (d) $24 \mathrm{~h}$.

$$
\Delta \psi=\int_{\sigma_{b}} \tilde{G}_{3 \mathrm{D}, m} \int_{0}^{t} J_{b} d \tau d \sigma_{b}=\int_{\sigma_{b}} t G_{3 \mathrm{D}, m} \bar{J}_{b} d \sigma_{b}
$$

where $\tilde{G}_{3 \mathrm{D}, m}$ is the value of $\tilde{G}_{3 \mathrm{D}}$ at a particular time between 0 and $t$ depending on the distribution of $J_{b}$. Because $H_{3 \mathrm{D}}$ is different from $t G_{3 \mathrm{D}, M}$, Eqs. (55) and (57) are different. If both $\tilde{G}_{3 \mathrm{D}}$ and $J_{b}$ are sign indefinite, the first mean value theorem cannot be applied to Eq. (47).

Equation (28) can be employed to illustrate numerically the uncertainties associated with this approach by comparing the $\psi$ computed from $H_{1 D} \overline{\mathscr{J}}_{b}$ with $\psi_{\text {true }}$ - the "true" mixing ratio computed from Eq. (28) over different time periods. Figure $6 \mathrm{a}, 6 \mathrm{c}$, and $6 \mathrm{~d}$ are $\psi$ computed using the averaged $\mathscr{J}_{b}$ over 6,12 , and $18 \mathrm{~h}$, respectively. The corresponding differences between $\psi$ and $\psi_{\text {true }}$ are shown in Figs. 6b, 6d, and 6e. It can be seen that when the surface flux is constant during the average period $[0 \mathrm{~h}, 6 \mathrm{~h}]$, the computed mixing ratio matches the true value. However, when the surface flux is sign indefinite during the averaged period $[0 \mathrm{~h}, 12 \mathrm{~h}]$ and $[0 \mathrm{~h}, 18 \mathrm{~h}]$, the differences between the computed mixing ratio and the true value are very large, and can reach as much as 20 ppmv at $30 \mathrm{~m}$ in the 
early morning. The big differences suggest that using Eq. (55) can introduce big errors in inverse modeling especially when surface flux is sign indefinite.

In the second approach the averaged observations are used as constraints. In this approach it is assumed that the time-averaged mixing ratio can be calculated by the product of the averaged transport operator and surface flux, namely,

$$
\Delta \bar{\psi}^{\prime}=\int_{\sigma_{b}} \bar{H}_{3 \mathrm{D}}(t) \bar{J}_{b} d \sigma_{b} .
$$

To examine this assumption, we first consider the case in which $J_{b}$ is sign indefinite and $\tilde{G}_{3 \mathrm{D}}$ is sign definite. In this case the averaged concentration is computed through the following equation:

$$
\begin{aligned}
\Delta \bar{\psi} & =\frac{1}{T} \int_{\sigma_{b}} \int_{0}^{T} \int_{0}^{t} \tilde{G}_{3 \mathrm{D}} J_{b}(\tau) d \tau d t d \sigma_{b} \\
& =\frac{1}{T} \int_{\sigma_{b}} \int_{0}^{T} J_{b, m}(t) \int_{0}^{t} \tilde{G}_{3 \mathrm{D}}(z, \tau) d t d \tau d \sigma_{b} \\
& =\int_{\sigma_{b}} J_{b, M} \bar{H} d \sigma_{b},
\end{aligned}
$$

where $J_{b, m}$ and $J_{b, M}$ are the value of $J_{b}$ at a particular time between 0 and $t$ and between 0 and $T$, respectively, and their values depend on the distribution of $\tilde{G}_{3 \mathrm{D}}$. Because $J_{b, M}$ is not the averaged surface flux, the averaged form of Eq. (47) cannot be put into Eq. (59). This is also true in other cases in which $J_{b}$ is sign definite, or both $J_{b}$ and $\tilde{G}_{3 \mathrm{D}}$ are sign indefinite.

The errors associated with this approach can be examined numerically by using Eq. (28) by comparing the difference between the averaged mixing ratio $\bar{\psi}_{\text {true }}$ computed from Eq. (28) and the estimated $\bar{\psi}$ from $\bar{H} \overline{\mathscr{J}}_{b}$. Figure 7 shows the variations of the true and estimated averaged variables over different time period with height. While the two are identical when the average period is $6 \mathrm{~h}$ during which the surface flux is constant, they are very different in the lower ABL when the average period is 12 and $18 \mathrm{~h}$ during which the surface flux is sign indefinite, and the differences can be as much as 7 ppmv. During the two average periods, the true mean mixing ratios are positive and the estimated ones are negative due to negative averaged surface fluxes over 12 and $18 \mathrm{~h}$. When the average period is $24 \mathrm{~h}$, the estimated mean mixing ratio is zero corresponding to zero mean surface flux.

The large relative errors mentioned above would have a significant impact on the accuracy of the estimated flux. In inverse modeling, the analysis error variance is the ratio of the product of background error variance and observation error variance to the sum of background error variance and observation error variance. Therefore, it increases with observation error variance for a given background error variance. When observation error variance is much larger than background error variance, the uncertainty in surface flux can be the same order of magnitude of observation error associated with the assumption about the separation of the GF and surface flux. However, the error in estimated surface flux would be much smaller during daytime during which surface flux is sign definite and the temporal variation of surface flux is small.

It should be pointed out that the errors shown above are associated with the use of time-mean surface flux in the concentration-surface flux equation. They are neither part of model errors nor the consequence of the rectifier effect contained in the numerical results. These errors would not be zero even if a numerical model is perfect or the rectifier effect is very weak.

\section{6. $\mathrm{CO}_{2}$ mixing ratio and $\mathrm{H}$ in the $1 \mathrm{D}$ diffusion equation with a nonseparable diffusion coefficient and time-varying ABL height}

In the above discussions, the diffusion coefficients have the simple forms and the variations of $K_{\psi}$ with time and height are separated, and the ABL height is a constant. To see if the above conclusions also hold in the more general case, the diffusion coefficient proposed by Holtslag and Boville (1993) using the $K$-profile closures will be employed to examine the variation of the $\mathrm{CO}_{2}$ mixing ratio, flux and the properties of the transport operator. The proposed diffusion coefficient has the following form

$$
K_{\psi}(z, t)=k w_{t} z\left[1-\frac{z}{h(t)}\right]^{2}
$$

where $k=0.4$ is the von Kármán constant, $w_{t}$ is the characteristic turbulent velocity scales, and $h(t)$ is the time-dependent depth of the ABL. For a give $h, K_{\psi}$ has a maximum $4 k w_{t} h / 27$ at $z=h / 3$. The nonlocal $K_{\psi}$ differs from the diffusion coefficients discussed in previous sections in that the variations of $K_{\psi}$ in time and height are no longer separable, and $h$ is time dependent.

In the following numerical calculations, a sinusoidal variation of $h$ between 6 and $18 \mathrm{~h}$ is used: $h$ increases from $100 \mathrm{~m}$ at $6 \mathrm{~h}$, reaches the maximum $1100 \mathrm{~m}$ at noon, and then decreases to $100 \mathrm{~m}$ at $18 \mathrm{~h}$. Corresponding to the variation of $h$, the magnitude of the vertical profile of $K_{\psi}$ also increases from $6 \mathrm{~h}$ and reaches the maximum at noon. In Holtslag and Boville (1993), $w_{t}$ is a complicated function of time and $z$, and has different forms 

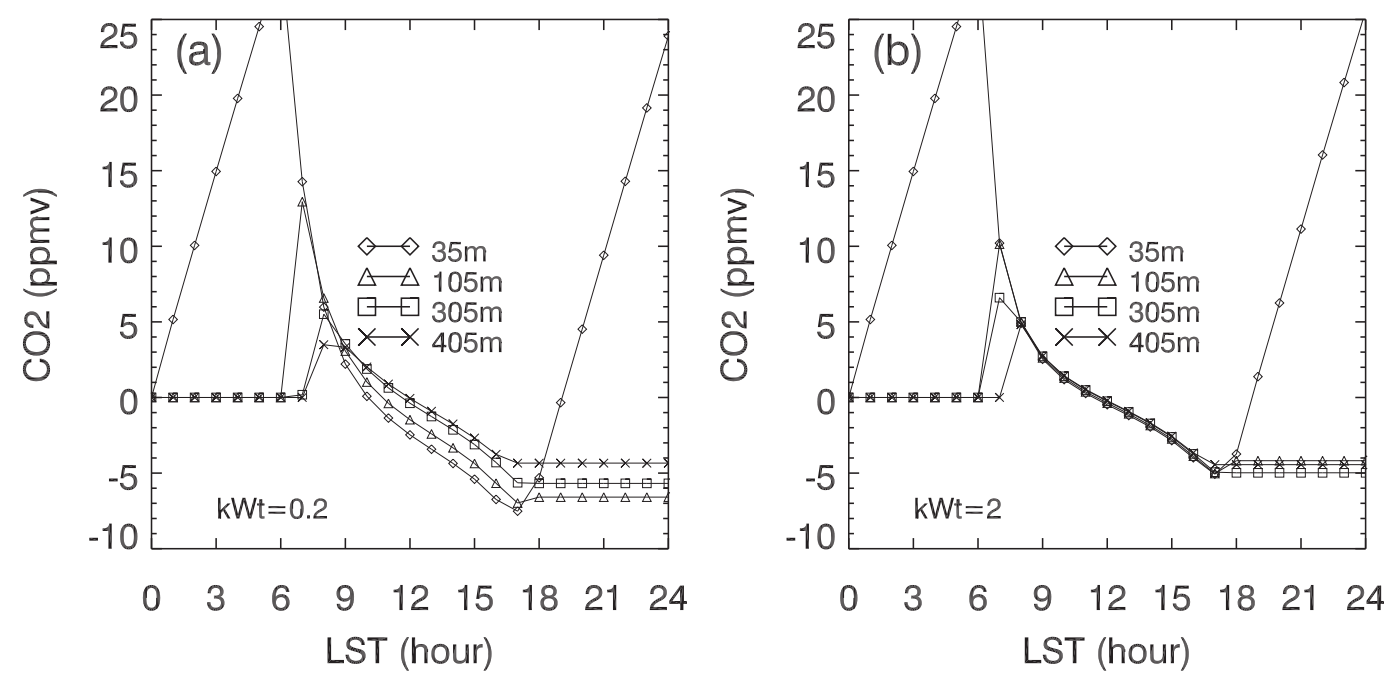

FIG. 8. Diurnal variation of the $\mathrm{CO}_{2}$ mixing ratio under the influence of the nonseparable diffusion coefficient defined in Eq. (59) and time-varying ABL height at different heights with (a) $k w_{t}=0.2$ and (b) $k w_{t}=2$.

under stable and unstable surface conditions. In our calculation, it is assumed that $w_{t}$ is a constant and neither the surface layer nor the entrainment zone is included for simplicity. Small and large values of $w_{t}$ are used to represent the stable and unstable condition, respectively. In computing $\psi$ and $H$, Eq. (6) is used as the lower boundary condition with the surface flux defined in Eq. (41). The upper boundary condition is $d \psi / d z=0$ when $z>h$, which is time dependent.

The variations of the $\mathrm{CO}_{2}$ mixing ratio with time at different height with $k w_{t}=0.2$ (stable case in which the convective velocity $w_{*}$ is comparable to the friction velocity $u_{*} \approx 0.5 \mathrm{~m} \mathrm{~s}^{-1}$ ) and $k w_{t}=2$ (unstable case in which $w_{*}=10 u_{*}$ ) are shown in Figs. $8 \mathrm{a}$ and 8b, respectively. Comparison between the two figures shows that while in both cases there is a jump in the $\mathrm{CO}_{2}$ mixing ratio in the early morning and evening at the low level $(30 \mathrm{~m})$, the vertical distribution of the $\mathrm{CO}_{2}$ mixing ratio in the stable case is less uniform than that in the unstable case due to weak vertical mixing. The comparison between Figs. 2 and 8 suggests that the variation of the $\mathrm{CO}_{2}$ mixing ratio calculated with the nonlocal scheme is less sensitive to the magnitude of $K_{\psi}$. While the mixing ratios above $100 \mathrm{~m}$ increase with time after $18 \mathrm{~h}$ in Fig. 2, they remain unchanged after $17 \mathrm{~h}$ in Fig. 8 due to the decrease of the ABL height to $100 \mathrm{~m}$ after $18 \mathrm{~h}$.

Contrary to the monotonic variation of the $\mathrm{CO}_{2}$ mixing ratio with height, the numerical results show that the variation of $\mathrm{CO}_{2}$ flux with height is not monotonic (not shown), similar to the vertical distribution of the flux in the case with the time-varying diffusion coefficient.

The temporal variations of the transport operator $H$ over $24 \mathrm{~h}$ at different heights with $k w_{t}=0.2$ and 2 are shown in Figs. 9a and 9c, respectively. The evolution pattern of $H$ is very similar to that of the $\mathrm{CO}_{2}$ mixing ratio shown in Fig. 8 suggesting that $H$ has a major contribution to the rectifier effect. The variations of $H$ with height at 12 and $24 \mathrm{~h}$ are shown in Figs. $9 \mathrm{~b}$ and $9 \mathrm{~d}$, for $k w_{t}=0.2$ and 2, respectively. It can be seen from the two figures that $H$ at hour 24 drops rapidly with height at around $z=100 \mathrm{~m}$ from 170 to 50 , much faster than the drop of $H$ in the time-varying diffusion coefficient case, and then gradually decreases to 20 below the top of ABL. However, $H$ at $12 \mathrm{~h}$ has very little change due to strong vertical mixing. Like the $\mathrm{CO}_{2}$ mixing ratio, $H$ is less sensitive to $K_{\psi}$ in this case than in the case with the time-varying $K_{\psi}$ shown in Figs. $5 \mathrm{~b}$ and $5 \mathrm{~d}$.

As with the case with the time-varying diffusion coefficient, uncertainties associated the concentrationmean surface flux equation can be examined by comparing the true mixing ratio and the mixing ratio computed from $H \overline{\mathscr{J}}_{b}$ when each individual observation is used (first approach), or by comparing the averaged true mixing ratio and $\bar{H} \overline{\mathscr{J}}_{b}$ when the averaged observations are used (second approach).

To validate the concentration-mean surface flux equation used in the first approach, the estimated mixing ratios at several levels are computed from $H \overline{\mathscr{J}}_{b}$ with $k w_{t}=2$ and presented along with their differences from the true mixing ratios in Fig. 10. As with the timevarying diffusion coefficient case, the estimated mixing ratios are identical to the true values when the average period is $6 \mathrm{~h}$ during which the surface flux is constant. However they are very different from the true values in the morning in the lower ABL when the average period is 12 and $18 \mathrm{~h}$ during which the surface flux is sign 

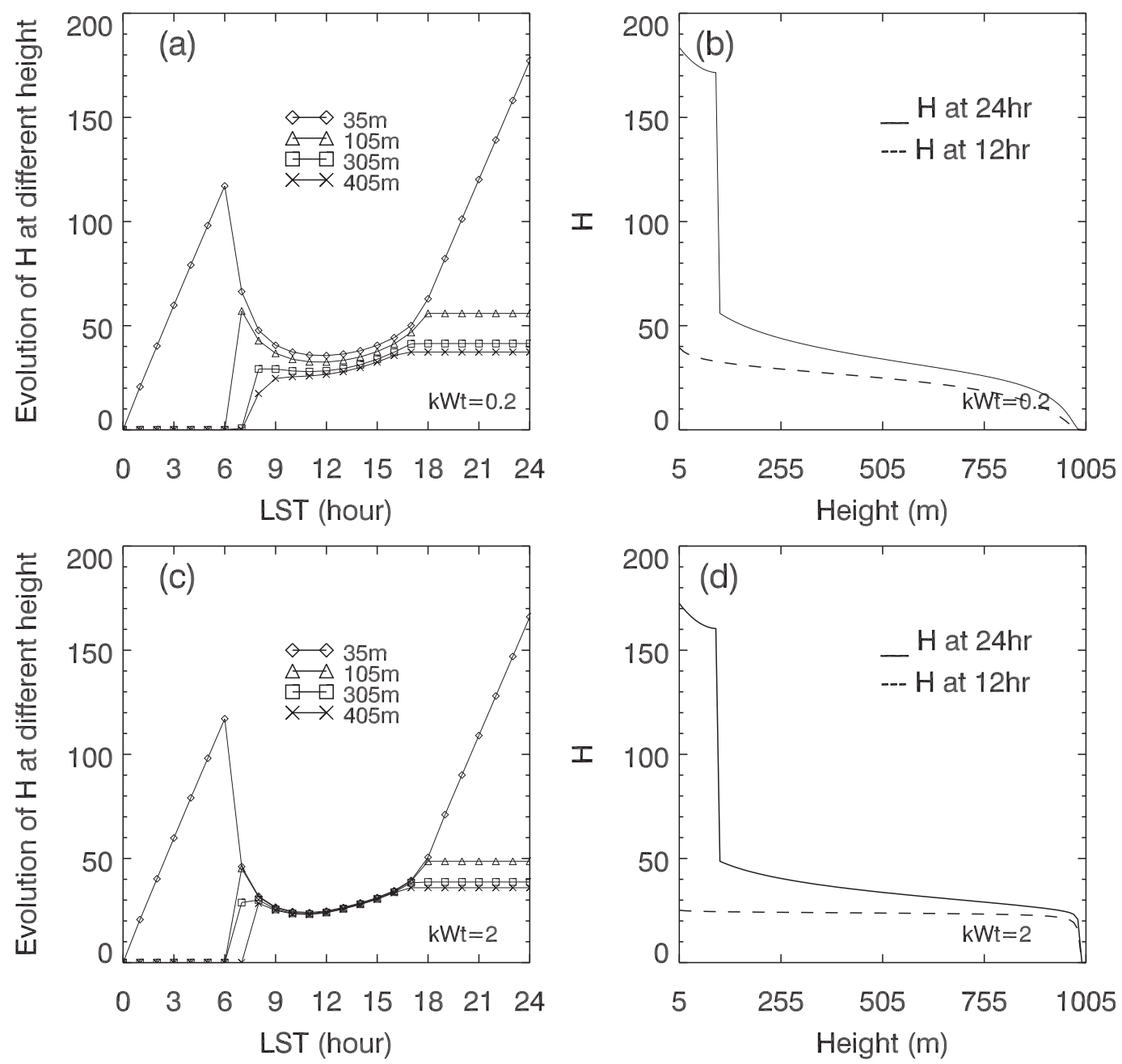

FIG. 9. Variation of $H$ under the diffusion coefficient defined by Eq. (60) and time-varying ABL height with time at different heights with (a) $k w_{t}=0.2$ and (c) $k w_{t}=2$, and the variation of $H$ at $t=12$ (dashed lines) and $t=24 \mathrm{~h}$ (solid lines) with height with (b) $k w_{t}=0.2$ and (d) $k w_{t}=2$.

indefinite. The differences can be more than $30 \mathrm{ppmv}$ at around $6 \mathrm{~h}$.

The concentration-mean surface flux equation used in the second approach can be validated in a similar way by computing the estimated time-mean mixing from $\bar{H} \overline{\mathscr{J}}_{b}$ with different average period. The variations of the estimated and true time-mean mixing ratios are presented in Fig. 11. Again, as with the time-varying diffusion coefficient case, the estimated and true mean mixing ratios are identical when the average period is $6 \mathrm{~h}$, but very different $(10 \mathrm{ppmv})$ for other average periods in the lower ABL. They have different sign when the average period is 18 and $24 \mathrm{~h}$.

Above numerical results show that although the nonseparable diffusion coefficient is more sophisticated than the those used in deriving the analytical solution, and the ABL height varies with time, the temporal and spatial distributions of mixing ratio, flux and transport operator shown in this section are very similar to those based on the analytical solutions. The agreement suggests that the analytical solutions capture the important features of the variations of passive tracers, and can describe the impacts of diffusivity on the evolution of the $\mathrm{CO}_{2}$ concentration, flux, and transport operator.

\section{Conclusions}

To understand the transport of passive tracers in the $\mathrm{ABL}$, the solutions to the 3D transport equation are derived to show explicitly the dependence of the concentration of passive tracers on boundary fluxes, nonlocal effect, and the GF within the domain and at the horizontal and vertical boundaries. This solution is employed in this work for understanding the connection 

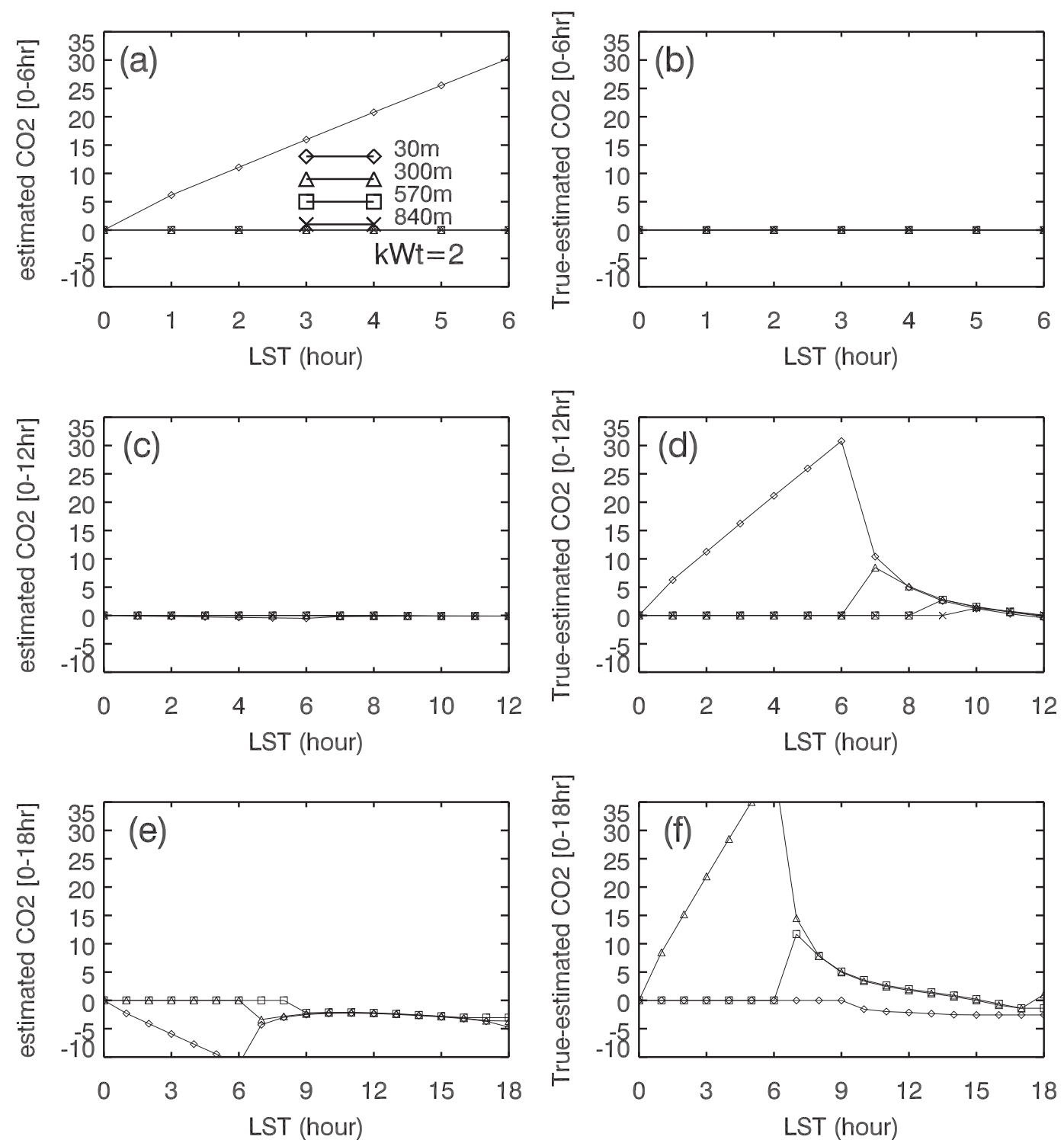

FIG. 10. The mixing ratios at four levels computed from $H \overline{\mathscr{J}}_{b}$ with $k w_{t}=2$ and with the average period of (a) 6, (c) 12, and (e) $18 \mathrm{~h}$. (b),(d),(f) Corresponding differences between the true and estimated mixing ratios.

between the concentrations of passive tracers in the atmosphere and the vertical fluxes in global inverse modeling.

The analytical solution to the 1D diffusion equation with time-varying diffusion coefficient is also derived to show the dependence of the concentration on height, time, diffusivity, and surface flux. This solution is applied to discuss the impacts of diffusivity on the diurnal variation of $\mathrm{CO}_{2}$ concentration, flux, the properties of the transport operator, and uncertainties associated with the assumptions underlying the concentration-mean flux equation. In addition, the analytical diffusion equation with the time-varying and slowly height-varying diffusion coefficient is derived by using the WKBJ method. The analytical solution shows that not only the value of diffusion coefficient but also its vertical variation can have an impact on the mixing ratio. By coupling with the nonlocal effect $\gamma_{\psi}$, the first derivative of the diffusion coefficient inserts an external force on the evolution of concentration. The value of first derivative at bottom enhances passive tracer concentrations, and the value within the domain reduces concentrations in the lower half of the ABL, but enhances concentrations in the upper half of the ABL. The second derivative leads to the exponential increase of mixing ratio in the whole domain. 

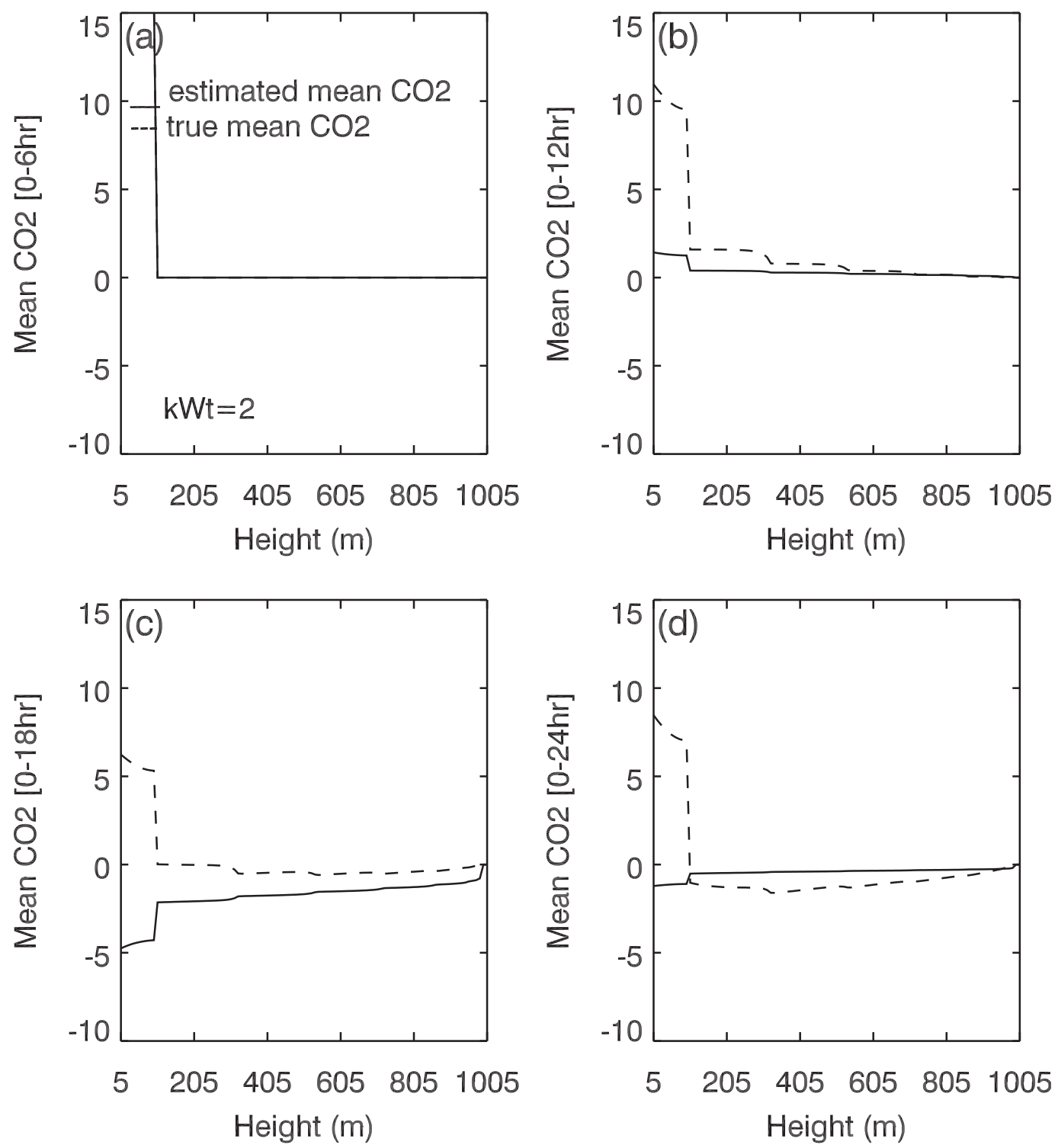

FIG. 11. Variations of the estimated time-mean $\mathrm{CO}_{2}$ mixing ratio computed from $\bar{H} \overline{\mathscr{J}}_{b}$ (dashed lines) and the true values (solid lines) with height over (a) 6, (b) 12, (c) 18, and (d) $24 \mathrm{~h}$.

The influence of diffusivity on the rectifier effect is examined based on the analytical solution to the $1 \mathrm{D}$ diffusion model in a semi-infinite domain. It is found that the variation of the $\mathrm{CO}_{2}$ mixing ratio depends on the diffusion coefficient through the GF, which is a highly nonlinear function of diffusion coefficient. The properties of GF, along with the observed surface flux, can describe the observed features of the diurnal variation of the $\mathrm{CO}_{2}$ mixing ratio in the $\mathrm{ABL}$, including 1) the increase of the biospheric $\mathrm{CO}_{2}$ mixing ratio in the lower $\mathrm{ABL}$ in the early morning, 2) rapid variation of the mixing ratio around $t=6 \mathrm{~h}$, and 3 ) the nearly homogeneous vertical distribution of the mixing ratio during the daytime. In addition, the impact of diffusivity on the $\mathrm{CO}_{2}$ flux is also examined. The results show that although the $\mathrm{CO}_{2}$ flux and $\mathrm{CO}_{2}$ mixing ratio have a similar relationship with diffusion coefficient, they have different vertical distribution. Unlike the $\mathrm{CO}_{2}$ mixing ratio, which decreases monotonically with increasing height, the magnitude of $\mathrm{CO}_{2}$ flux can actually increase with height depending on the diffusion coefficient, height and time. The results also show that the $\mathrm{CO}_{2}$ flux has a less uniformly vertical distribution than the $\mathrm{CO}_{2}$ mixing ratio during the daytime.

In this work, the properties of the $1 \mathrm{D}$ transport operator explored. It is found that although the transport 
operator at a given time $t$ is the accumulative contribution of GF prior to $t$, only the GF close to $t$ has a major contribution. This property justifies the reduction of the size of the matrix of the transport operator in inverse modeling. The results suggest that the transport operator makes a major contribution to the rectifier effect observed in the lower ABL. Since the transport operator is associated with dynamic processes, it is fundamentally different from the interpolation operator used in the conventional data assimilation schemes. Due to the exponential decrease of GF with height, transport operator also decreases as height increases and consequently leads to the decrease of the Kalman gain. As the magnitude of Kalman gain describes the strength of the constraints of observations on the estimated surface flux, the results suggest that surface observations impose stronger constraints than observations of aircraft and satellites at high altitude on the estimated surface flux in inverse modeling.

The analytical solutions, along with the first mean value theorem for integration, are employed to discuss the uncertainties associated with the two kinds of concentration-mean surface flux equation which can be used in inverse modeling to connect the $\mathrm{CO}_{2}$ mixing ratio in the atmosphere and the averaged surface flux to be estimated. While the time-varying transport operator is used to sample the model values at each individual observation site and time in the first kind of equation, the time-averaged transport operator is used in the second kind. The uncertainties associated with the two equations are discussed based on the analytical transport operator and the solution of the $1 \mathrm{D}$ diffusion model. Both analytical and numerical results show that both approaches may introduce big errors (10 ppmv, or $50 \%$ relative error) in the lower $\mathrm{ABL}$ when the surface flux is sign indefinite during the average period. These errors would lead to the same order of magnitude error in the estimated surface flux if the observation error were much larger than the background error.

The numerical solution to the $1 \mathrm{D}$ diffusion model with a more complicated nonseparable diffusion coefficient and the variable ABL height also shows similar impacts of diffusivity on the spatial and temporal variation of the $\mathrm{CO}_{2}$ mixing ratio, flux, and transport operator. All these results suggest that most of the conclusions about the features of variation of passive tracers and the impacts of diffusivity on tracer's concentration, flux and transport operator based on the analytical solution also hold in more general cases.

Acknowledgments. The author wishes to thank Drs. C. Stroud, E. Chan, D. Chan, and S. Ralf for their comments and proofreading the manuscript. Comments and suggestions from three anonymous reviewers are greatly appreciated.

\section{APPENDIX A}

\section{Solution to the 3D Transport Equation of Passive Tracers}

By letting $t \rightarrow \tau$ and $\mathbf{r} \rightarrow \mathbf{r}^{\prime}$, Eq. (6) can be put into the following form:

$\frac{\partial \psi}{\partial \tau}+\mathbf{u}_{0} \cdot \nabla_{0} \psi-\frac{\partial}{\partial z^{\prime}}\left[K_{\psi}\left(\mathbf{r}^{\prime}, \tau\right) \frac{\partial \psi}{\partial z^{\prime}}\right]=\Upsilon-\gamma_{\psi} \frac{\partial K_{\psi}}{\partial z^{\prime}}$

Multiplying Eq. (A1) by $G_{3 \mathrm{D}}$ and Eq. (11) by $\psi$, and subtracting the two equations, one obtains

$$
\begin{aligned}
& \frac{\partial\left(G_{3 \mathrm{D}} \psi\right)}{\partial \tau}+\nabla_{0} \cdot\left(G_{3 \mathrm{D}} \psi \mathbf{u}_{0}\right)-G_{3 \mathrm{D}} \frac{\partial}{\partial z^{\prime}}\left(K_{\psi} \frac{\partial \psi}{\partial z^{\prime}}\right)+\psi \frac{\partial}{\partial z^{\prime}}\left(K_{\psi} \frac{\partial G_{3 \mathrm{D}}}{\partial z^{\prime}}\right) \\
& =\left(\Upsilon-\gamma_{\psi} \frac{\partial K_{\psi}}{\partial z^{\prime}}\right) G_{3 \mathrm{D}}-\delta\left(\mathbf{r}-\mathbf{r}^{\prime}\right) \delta(t-\tau) \psi .
\end{aligned}
$$

Integrating Eq. (A2) with respect to $\mathbf{r}^{\prime}$ in the whole domain and respect to $\tau$ from 0 to $t+\varepsilon_{0}$, where $\varepsilon_{0}$ is a small positive number yields

$$
\begin{aligned}
\psi(\mathbf{r}, t)= & \int_{\tau=0}^{\tau=t+\varepsilon_{0}} \int_{\Omega}\left[G_{3 \mathrm{D}} \frac{\partial}{\partial z^{\prime}}\left(K_{\psi} \frac{\partial \psi}{\partial z^{\prime}}\right)-\psi \frac{\partial}{\partial z^{\prime}}\left(K_{\psi} \frac{\partial G_{3 \mathrm{D}}}{\partial z^{\prime}}\right)\right] d \tau d^{3} \mathbf{r}^{\prime} \\
& -\sum_{i=1}^{4} \int_{\sigma_{v, i}} \int_{\tau=0}^{\tau=t+\varepsilon_{0}} \psi G_{h i} \mathbf{u}_{0 i} d \sigma_{v, i}+\int_{\tau=0}^{\tau=t+\varepsilon_{0}} \int_{\Omega} G_{3 \mathrm{D}}\left(\Upsilon-\gamma_{\psi} \frac{\partial K_{\psi}}{\partial z^{\prime}}\right) d \mathbf{r}^{\prime} d \tau-\left.\int_{\Omega}\left(G_{3 \mathrm{D}} \psi\right)\right|_{\tau=0} ^{\tau=t+\varepsilon_{0}}
\end{aligned}
$$


Using the boundary conditions of $G_{3 \mathrm{D}}$ and $\psi$, the causality relation $G_{3 \mathrm{D}}\left(\mathbf{r}, t \mid \mathbf{r}^{\prime}, t+\varepsilon_{0}\right)$ and letting $\varepsilon_{0}=0$, one obtains the solution to the $3 \mathrm{D}$ transport equation for passive tracers [Eq. (13)].

\section{APPENDIX B}

\section{Transformation of Eq. (29)}

To remove the term associated with $\partial \psi / \partial z$ in Eq. (35), we use the transformation $\psi=\eta(z) \phi(z, t)$ with which Eq. (29) can be written as

$$
\begin{aligned}
\frac{\partial \phi}{\partial \hat{t}}= & D \frac{\partial^{2} \phi}{\partial z^{2}}+\frac{1}{\eta}\left(2 D \frac{d \eta}{d z}+\frac{d D}{d z} \eta\right) \frac{\partial \phi}{\partial z} \\
& +\frac{1}{\eta}\left(D \frac{d^{2} \eta}{d z^{2}}+\frac{d D}{d z} \frac{d \eta}{d z}\right) \phi-\frac{\gamma_{s}}{\eta} \frac{d D}{d z} .
\end{aligned}
$$

After eliminating the coefficient of $d \phi / d z$ by letting $2 D \eta_{z}+D_{z} \eta=0$ one has

$$
\begin{aligned}
\eta & =\frac{1}{\sqrt{D}} \\
\frac{1}{\eta}\left(D \frac{d^{2} \eta}{d z^{2}}+\frac{d D}{d z} \frac{d \eta}{d z}\right) & =\frac{1}{4 D}\left(\frac{d D}{d z}\right)^{2}-\frac{1}{2} \frac{d^{2} D}{d z^{2}} \equiv \Gamma .
\end{aligned}
$$

Thus, Eq. (B1) can be transformed into the following equation:

$$
\frac{\partial \phi}{\partial \hat{t}}=D \frac{\partial^{2} \phi}{\partial z^{2}}+\Gamma \phi-\gamma_{\psi} \sqrt{D} \frac{d D}{d z}
$$

\section{APPENDIX C}

\section{The Solution to Eq. (32)}

Applying the Laplace transform to Eq. (32) yields

$$
\frac{d^{2} \hat{\Phi}}{d z^{2}}-\Gamma_{1}^{2} \hat{\Phi}=\frac{\gamma_{s}(p)}{\sqrt{D}} \frac{d D}{d z},
$$

where $\Gamma_{1}=\sqrt{p / D}>0$ and $\gamma_{s}$ is Laplace transform of $\gamma_{\psi} \exp (-\Gamma \hat{t})$.

Applying the Laplace transform of the lower and upper boundary conditions [Eqs. (16a) and (16b)], one obtains

$$
\frac{\partial \hat{\Phi}}{\partial z}=-\frac{\mathscr{F}_{t}(p)}{\sqrt{D\left(z_{T}\right)}}+\left.\frac{D_{z}}{2 D}\right|_{z=z_{T}} \hat{\Phi}
$$

$$
\frac{\partial \hat{\Phi}}{\partial z}=-\frac{\mathscr{F}_{b}(p)}{\sqrt{D_{0}}}+\left.\frac{D_{z}}{2 D}\right|_{z=0} \hat{\Phi}
$$

where $\mathscr{F}_{t}$ and $\mathscr{F}_{b}$ are the Laplace transform of $\mathscr{J}_{t} \exp (-\Gamma \hat{t}) / T$ and $\mathscr{J}_{b} \exp (-\Gamma \hat{t}) / T$, respectively.

By using the WKBJ method, the approximate solution to Eq. (C1) (including solution to the homogeneous and particular solution) can be written as

$$
\begin{aligned}
\hat{\Phi}(z, p)= & \frac{a}{\sqrt{\Gamma_{1}}} \exp [K(z) \sqrt{p}]+\frac{b}{\sqrt{\Gamma_{1}}} \exp [-K(z) \sqrt{p}] \\
& +\zeta(z, p),
\end{aligned}
$$

where $\zeta$ is the particular solution, $a$ and $b$ are constants to be determined in appendix $\mathrm{D}$, and

$$
K(z)=\int^{z} \frac{d z^{\prime}}{\sqrt{D\left(z^{\prime}\right)}}
$$

Applying inverse Laplace transform to Eq. (C4), one obtains

$$
\begin{aligned}
\psi(z, t) & =\eta \phi=\eta \exp (\Gamma \hat{t}) L^{-1} \hat{\Phi} \\
& =\lambda\left[\int_{0}^{t} \mathscr{J}_{b}(\tau) \mathscr{G}_{b} d \tau-\int_{0}^{t} \mathscr{J}_{t}(\tau) \mathscr{G}_{t} d \tau\right]+\varepsilon \int_{0}^{t} \gamma_{\psi} \mathscr{G} d \tau,
\end{aligned}
$$

where

$$
\begin{array}{rlr}
\mathscr{G}_{b} & =I_{b}(z, \mathscr{T}) \exp (\Gamma \mathscr{T}), & (\mathrm{C} 6) \\
\mathscr{G}_{t} & =I_{t}(z, \mathscr{T}) \exp (\Gamma \mathscr{T}), \\
I_{b}(z, \mathscr{T}) & =\sum_{n=-\infty}^{n=\infty} \frac{1}{\sqrt{D \pi \mathscr{T}}} \exp \left\{-\frac{\left[2 n K_{0}\left(z_{T}\right)-K_{0}(z)\right]^{2}}{4 D_{0} \mathscr{T}}\right\},
\end{array}
$$

$$
I_{t}(z, \mathscr{T})=\sum_{n=-\infty}^{n=\infty} \frac{1}{\sqrt{D \pi \mathscr{T}}}\left\{\frac{-\left[K_{0}(z)+(2 n-1) K_{0}\left(z_{T}\right)\right]^{2}}{4 D_{0} \mathscr{T}}\right\},
$$

$$
\begin{aligned}
\mathscr{G}= & -\mathscr{E}_{0} \lambda \int_{0}^{t-\tau} I_{b t}\left(z, \mathscr{T}^{\prime}\right) T\left(\tau^{\prime}\right) T(\tau) \exp (\Gamma \mathscr{T}) d \tau^{\prime} \\
& +\mathscr{E}_{1}(z) T(\tau) \exp (\Gamma \mathscr{T}) \\
I_{b t}\left(z, \mathscr{T}^{\prime}\right)= & I_{b}\left(z, \mathscr{T}^{\prime}\right)+I_{t}\left(z, \mathscr{T}^{\prime}\right) \\
\mathscr{T}^{\prime}= & \int_{\tau^{\prime}}^{t-\tau} T\left(\tau^{\prime \prime}\right) d \tau^{\prime \prime}
\end{aligned}
$$




$$
K_{0}(z)=\int^{z}\left[1+\varepsilon \frac{z^{\prime}}{z_{T}}\left(1-\frac{z^{\prime}}{z_{T}}\right)\right]^{-1 / 2} d z^{\prime}
$$

where $\mathscr{E}_{1}(z)$ is defined in Eq. (D6) and $\mathscr{E}_{0}=\mathscr{E}(0)=$ $D_{0}^{3 / 2} / z_{T}$. In deriving Eq. (C10) the following equation is used:

$$
L^{-1}\left[\frac{H_{1}(p) H_{2}(p)}{p}\right]=\int_{0}^{t} \int_{0}^{t-\tau} h_{1}(\tau) h_{2}\left(\tau^{\prime}\right) d \tau^{\prime} d \tau,
$$

where $H_{1}(p)=L h_{1}$ and $H_{2}(p)=L h_{2}$.

\section{APPENDIX D}

\section{Solution to Eq. (C1)}

To obtain the particular solution to Eq. (C1), we expand $\zeta$ and $p / D$ as

$$
\begin{gathered}
\zeta=\zeta_{0}+\varepsilon \zeta_{1}+\varepsilon^{2} \zeta_{2}+\ldots, \\
\frac{p}{D(z)} \approx \frac{p}{D_{0}}\left[1-\varepsilon \frac{z}{z_{T}}\left(1-\frac{z}{z_{T}}\right)\right] .
\end{gathered}
$$

Substituting Eqs. (D1) and (D2) into Eq. (C1) yields

$$
\frac{d^{2} \zeta_{0}}{d z^{2}}-\frac{p}{D_{0}} \zeta_{0}=0, \quad \Rightarrow \quad \zeta_{0}=0
$$

at the leading order. At the second order

$$
\frac{d^{2} \zeta_{1}}{d z^{2}}-\frac{p}{D_{0}} \zeta_{1}=\frac{\gamma_{s}(p) \sqrt{D_{0}}}{z_{T}}\left(1-\frac{2 z}{z_{T}}\right)
$$

from which one has

$$
\zeta_{1}=-\frac{\gamma_{s}(p) D_{0}^{3 / 2}}{p z_{T}}\left(1-\frac{2 z}{z_{T}}\right)
$$

Thus, the particular solution of Eq. (C1) up to the second order is

$$
\zeta(z, p)=-\varepsilon \gamma_{s}(p) \frac{D_{0}^{3 / 2}}{p z_{T}}\left(1-\frac{2 z}{z_{T}}\right) \equiv \varepsilon \gamma_{s}(p) \frac{\mathscr{E}_{1}(z)}{p},
$$

from which the complete solution can be put as

$$
\begin{aligned}
\hat{\Phi}(z, p)= & \frac{a}{\sqrt{\Gamma_{1}}} \exp [K(z) \sqrt{p}]+\frac{b}{\sqrt{\Gamma_{1}}} \exp [-K(z) \sqrt{p}] \\
& +\varepsilon \frac{\gamma_{s}(p) \mathscr{E}_{1}(z)}{p} .
\end{aligned}
$$

To use the boundary conditions, we first compute

$$
\begin{aligned}
\frac{\partial \hat{\Phi}}{\partial z}-\frac{D_{z}}{2 D} \hat{\Phi}= & \frac{a}{\sqrt{\Gamma_{1}}}\left(-\frac{D_{z}}{4 D}+\Gamma_{1}\right) \exp (K \sqrt{p}) \\
& +\frac{b}{\sqrt{\Gamma_{1}}}\left(-\frac{D_{z}}{4 D}-\Gamma_{1}\right) \exp (-K \sqrt{p}) \\
& +\varepsilon \frac{\gamma_{s}(p) \mathscr{E}_{1}(z)}{p}
\end{aligned}
$$

Because $D(z=0)=D\left(z_{T}\right)=D_{0}$, the values of $\Gamma$ and $D_{z} / 4 D$ at boundaries can be obtained as

$$
\Gamma_{1}(z=0)=\Gamma_{1}\left(z_{T}\right)=\sqrt{p / D_{0}} \equiv \Gamma_{0}
$$

and

$$
\left.\frac{D_{z}}{4 D}\right|_{z=0}=-\left.\frac{D_{z}}{4 D}\right|_{z_{T}}=\frac{\varepsilon}{4 z_{T}} \equiv \varepsilon_{1}
$$

Substituting Eq. (D8) into boundary conditions Eqs. (C2a) and (C2b) and using Eqs. (D9) and (D10) yields

$$
\begin{aligned}
& \frac{a}{\sqrt{\Gamma_{0}}}\left(\varepsilon_{1}+\Gamma_{0}\right) \exp \left[K\left(z_{T}\right) \sqrt{p}\right] \\
& \quad+\frac{b}{\sqrt{\Gamma_{0}}}\left(\varepsilon_{1}-\Gamma_{0}\right) \exp \left[-K\left(z_{T}\right) \sqrt{p}\right]=-\frac{\mathscr{F}_{t}}{\sqrt{D_{0}}} \\
& \quad-\varepsilon \frac{\gamma_{s}(p) \mathscr{E}_{1}\left(z_{T}\right)}{p},
\end{aligned}
$$

$$
\begin{aligned}
& \frac{a}{\sqrt{\Gamma_{0}}}\left(-\varepsilon_{1}+\Gamma_{0}\right)+\frac{b}{\sqrt{\Gamma_{0}}}\left(-\varepsilon_{1}-\Gamma_{0}\right)=-\frac{\mathscr{F}_{b}}{\sqrt{D_{0}}} \\
& \quad-\varepsilon \frac{\gamma_{s}(p) \mathscr{E}_{1}(0)}{p} .
\end{aligned}
$$

Since $\varepsilon_{1} \sim \mathscr{O}\left(\varepsilon^{2}\right)$, its contribution in Eqs. (D11a) and (D11b) can be ignored and $a$ and $b$ can be obtained as

$$
\begin{aligned}
a= & \frac{\mathscr{F}_{b} \exp \left[-K\left(z_{T}\right) \sqrt{p}\right]-\mathscr{F}_{t}}{2 \sqrt{\Gamma_{0} D_{0}} \sinh \left[K\left(z_{T}\right) \sqrt{p}\right]} \\
& -\varepsilon \gamma_{s} \mathscr{E}_{0} \frac{\exp \left[-K\left(z_{T}\right) \sqrt{p}\right]+1}{2 p \sqrt{\Gamma_{0} D_{0}} \sinh \left[K\left(z_{T}\right) \sqrt{p}\right]}, \\
b= & \frac{\mathscr{F}_{b} \exp \left[K\left(z_{T}\right) \sqrt{p}\right]-\mathscr{F}_{t}}{2 \sqrt{\Gamma_{0} D_{0}} \sinh \left[K\left(z_{T}\right) \sqrt{p}\right]} \\
& -\varepsilon \gamma_{s} \mathscr{E}_{0} \frac{\exp \left[K\left(z_{T}\right) \sqrt{p}\right]+1}{2 p \sqrt{\Gamma_{0} D_{0}} \sinh \left[K\left(z_{T}\right) \sqrt{p}\right]},
\end{aligned}
$$

where $\mathscr{E}_{0}=-D_{0}^{3 / 2} / z_{T}$. In deriving Eqs. (D12a) and (D12b), $\mathscr{E}_{0}=-\mathscr{E}\left(z_{T}\right)=-D_{0}^{3 / 2} / z_{T}$ are used. Thus, $\hat{\Phi}$ can be obtained as 


$$
\begin{aligned}
\hat{\Phi}= & \lambda \frac{\mathscr{F}_{b} \cosh \left\{\left[K\left(z_{T}\right)-K(z)\right] \sqrt{p}\right\}-\mathscr{F}_{t} \cosh [K(z) \sqrt{p}]}{\sqrt{p} \sinh \left[K\left(z_{T}\right) \sqrt{p}\right]} \\
& -\varepsilon \lambda \gamma_{s} \mathscr{E}_{0} \frac{\cosh \left\{\left[K\left(z_{T}\right)-K(z)\right] \sqrt{p}\right\}+\cosh [K(z) \sqrt{p}]}{p \sqrt{p} \sinh \left[\left(K\left(z_{T}\right) \sqrt{p}\right]\right.}+\varepsilon \frac{\gamma_{s} \mathscr{E}_{1}(z)}{p},
\end{aligned}
$$

where $\lambda=[D(z) / D(0)]^{1 / 4}$.

\section{REFERENCES}

Baker, I., A. Denning, N. Hanan, L. Prihodko, M. Uliasz, P. Vidale, K. Davis, and P. Bakwin, 2003: Simulated and observed fluxes of sensible and latent heat and $\mathrm{CO}_{2}$ at the WLEF-TV tower using SiB2.5. Global Change Biol., 9, 1262-1277, https:// doi.org/10.1046/j.1365-2486.2003.00671.x.

Bakwin, P., P. P. Tans, D. Hurst, and C. Zhao, 1998: Measurements of carbon dioxide on very tall towers: Results of the NOAA/ CMDL program. Tellus, 50B, 401-415, https://doi.org/10.3402/ tellusb.v50i5.16216.

Basha, H. A., and F. S. El-Habel, 1993: Analytical solution of the one-dimensional time-dependent transport equation. Water Resour. Res., 29, 3209-3214, https://doi.org/10.1029/ 93WR01038.

Batchelor, G., 2000: An Introduction to Fluid Dynamics. Cambridge University Press, 615 pp.

Bateman, H., 1954: Tables of Integral Transforms. Vol. 1, A. Erdelyi, Ed., McGraw-Hill, 391 pp.

Bruhwiler, L., A. Michalak, W. Peters, D. F. Baker, and P. Tans, 2005: An improved Kalman smoother for atmospheric inversions. Atmos. Chem. Phys., 5, 2691-2702, https://doi.org/ 10.5194/acp-5-2691-2005.

Butler, M., K. Davis, A. Denning, and S. Kawa, 2010: Using continental observations in global atmospheric inversions of $\mathrm{CO}_{2}$ : North American carbon sources and sinks. Tellus, 62B, 550-572, https://doi.org/10.1111/j.1600-0889.2010.00501.x.

Chen, B., J. M. Chen, J. Liu, D. Chan, K. Higuchi, and A. Shashkov, 2004: A vertical diffusion scheme to estimate the atmospheric rectifier effect. J. Geophys. Res., 109, D04306, https://doi.org/ 10.1029/2003JD003925.

Chen, J., and C. Liu, 2011: Generalized analytical solution for advection-dispersion equation in finite spatial domain with arbitrary time-dependent inlet boundary condition. Hydrol. Earth Syst. Sci., 15, 2471-2479, https://doi.org/10.5194/ hess-15-2471-2011.

Daley, D., 1993: Atmospheric Data Assimilation. Cambridge University Press, $457 \mathrm{pp}$.

Davis, K., P. Bakwin, C. Yi, B. Berger, C. Zhao, R. M. Teclaw, and J. G. Isenbrands, 2003: The annual cycles of $\mathrm{CO}_{2}$ and $\mathrm{H}_{2} \mathrm{O}$ exchange over a northern mixed forest as observed from a very tall tower. Global Change Biol., 9, 1278-1293, https:// doi.org/10.1046/j.1365-2486.2003.00672.x.

Denning, A. S., G. J. Collatz, C. Zhang, D. A. Randall, J. A. Berry, P. J. Sellers, G. D. Colello, and D. A. Dazlich, 1996a: Simulations of terrestrial carbon metabolism and atmospheric co2 in a general circulation model. Part 1: Surface carbon fluxes. Tellus, 48B, 521-542, https://doi.org/10.3402/ tellusb.v48i4.15930.

, D. A. Randall, G. J. Collatz, and P. J. Sellers, 1996b: Simulations of terrestrial carbon metabolism and atmospheric $\mathrm{CO}_{2}$ in a general circulation model. Part 2: Simulated $\mathrm{CO}_{2}$ concentrations. Tellus, 48B, 543-567, https://doi.org/ 10.3402/tellusb.v48i4.15931.

Fung, I., K. Prentice, E. Matthews, J. Lerner, and G. Russell, 1983: Three-dimensional tracer model study of atmospheric $\mathrm{CO}_{2}$ : Response to seasonal exchanges with the terrestrial biosphere. J. Geophys. Res., 88, 1281-1294, https://doi.org/10.1029/ JC088iC02p01281.

Gerritsen, K. O., 2012: Estimation of advective fluxes from $\mathrm{CO}_{2}$ flux profile observations at the Cabauw Tower. Tech. Rep. TR-330, Royal Netherlands Meteorological Institute, 72 pp., http://bibliotheek.knmi.nl/knmipubTR/TR330.pdf.

Gill, A. E., 1982: Atmosphere-Ocean Dynamics. Academic Press, $297 \mathrm{pp}$.

Gloor, M., S. M. Fan, S. W. Pacala, J. L. Sarmiento, and M. Ramonet, 1999: A model-based evaluation of inversions of atmospheric transport, using annual mean mixing ratios, as a tool to monitor fluxes of nonreactive trace substances like $\mathrm{CO}_{2}$ on a continental scale. J. Geophys. Res., 104, 14 245-14260, https://doi.org/10.1029/1999JD900132.

Greenberg, M., 1971: Application of Green's Functions in Science and Engineering. Prentice-Hall, $141 \mathrm{pp}$.

Gurney, K. R., and Coauthors, 2003: TransCom 3 inversion intercomparison: 1 . Annual mean control results and sensitivity to transport and prior flux information. Tellus, 55B, 555-579, https://doi.org/10.3402/tellusb.v55i2.16728.

— , and Coauthors, 2004: TransCom 3 inversion intercomparison: Model mean results for the estimation of seasonal carbon sources and sinks. Global Biogeochem. Cycles, 18, GB1010, https://doi.org/10.1029/2003GB002111.

Holtslag, A. A. M., and C. Moeng, 1991: Eddy diffusivity and countergradient transport in the convective atmospheric boundary layer. J. Atmos. Sci., 48, 1690-1698, https://doi.org/ 10.1175/1520-0469(1991)048<1690:EDACTI >2.0.CO;2.

___ , and B. A. Boville, 1993: Local versus non-local boundarylayer diffusion on a global climate model. J. Climate, 6, 1825-1842, https://doi.org/10.1175/1520-0442(1993)006<1825: LVNBLD $>2.0 . C O ; 2$.

Holzer, M., 1999: Analysis of passive tracer transport as modeled by an atmospheric general circulation model. J. Climate, $\mathbf{1 2}$, 1659-1684, https://doi.org/10.1175/1520-0442(1999)012<1659: AOPTTA $>2.0 . \mathrm{CO} ; 2$.

_ , and T. M. Hall, 2000: Transit-time and tracer-age distributions in geophysical flows. J. Atmos. Sci., 57, 3539-3558, https://doi.org/ 10.1175/1520-0469(2000)057<3539:TTATAD>2.0.CO;2.

Houweling, S., and Coauthors, 2010: The importance of transport model uncertainties for the estimation of $\mathrm{CO}_{2}$ sources and sinks using satellite measurements. Atmos. Chem. Phys., 10, 9981-9992, https://doi.org/10.5194/acp-10-9981-2010.

Hurwitz, M., D. Ricciuto, P. Bakwin, K. Davis, W. Wang, C. Yi, and M. Butler, 2004: Transport of carbon dioxide in the presence of storm systems over a northern Wisconsin forest. J. Atmos. Sci., 61, 607-618, https://doi.org/10.1175/ 1520-0469(2004)061<0607:TOCDIT>2.0.CO;2.

Jaiswal, D. K., A. Kumar, and R. R. Yadav, 2011: Analytical solution to the one-dimensional advection0diffusion equation with 
temporally dependent coefficients. J. Water Resource Prot., 3 , 76-84, https://doi.org/10.4236/jwarp.2011.31009.

Kretschmer, R., C. Gerbig, U. Karstens, and F.-T. Koch, 2012: Error characterization of $\mathrm{CO}_{2}$ vertical mixing in the atmospheric transport model WRF-VPRM. Atmos. Chem. Phys., 12, 2441-2458, https://doi.org/10.5194/acp-12-2441-2012.

Larson, V. E., and H. Volkmer, 2008: An idealized model of the one-dimensional carbon dioxide rectifier effect. Tellus, $\mathbf{6 0 B}$, 525-536, https://doi.org/10.1111/j.1600-0889.2008.00368.x.

Lee, X., 1998: On micrometeorological observations of surface-air exchange over tall vegetation. Agric. For. Meteor., 91, 39-49, https://doi.org/10.1016/S0168-1923(98)00071-9.

Leij, F. J., E. Priesack, and M. G. Schaap, 2000: Solute transport modeled with Green's functions with application to persistent solute sources. J. Contam. Hydrol., 41, 155-173, https:// doi.org/10.1016/S0169-7722(99)00062-5.

Lin, J. C., and C. Gerbig, 2005: Accounting for the effect of transport errors on tracer inversions. Geophys. Res. Lett., 32, L01802, https://doi.org/10.1029/2004GL021127.

Louis, J.-F., 1979: A parametric model of vertical eddy fluxes in the atmosphere. Bound.-Layer Meteor., 17, 187-202, https://doi.org/10.1007/BF00117978.

—, M. Tiedtkt, and J. F. Geleyn, 1982: A short history of the PBL parametrization at ECMWF. Proc. Workshop on Planetary Boundary Layer Parameterization, Shinfield Park, Reading, ECMWF, 59-79, https://www.ecmwf.int/node/10845.

Makar, P. A., R. Nissen, A. Teakles, J. Zhang, Q. Zheng, M. D. Moran, H. Yau, and C. diCenzo, 2014: Turbulent transport, emissions and the role of compensating errors in chemical transport models. Geosci. Model Dev., 7, 1001-1024, https://doi.org/10.5194/gmd-7-1001-2014.

Mellor, G., and T. Yamada, 1982: Development of a turbulence closure model for geophysical fluid problems. Rev. Geophys. Space Phys., 20, 851-875, https://doi.org/10.1029/RG020i004p00851.

O'Brien, J. J., 1970: A note on the vertical structure of the eddy exchange coefficient in the planetary boundary layer. J. Atmos. Sci., 27, 1213-1215, https://doi.org/10.1175/1520-0469(1970)027<1213: ANOTVS $>2.0 . \mathrm{CO} ; 2$.

Peters, W., and Coauthors, 2005: An ensemble data assimilation system to estimate $\mathrm{CO}_{2}$ surface fluxes from atmospheric trace gas observations. J. Geophys. Res., 110, D24304, https:// doi.org/10.1029/2005JD006157.
Peylin, P., D. Baker, J. Sarmiento, P. Ciais, and P. Bousquet, 2002: Influence of transport uncertainty on annual mean and seasonal inversions of atmospheric $\mathrm{CO}_{2}$ data. J. Geophys. Res., 107, 4385, https://doi.org/10.1029/2001JD000857.

Pino, D., J. Vilà-Guerau de Arellano, W. Peters, J. Schröter, C. C. van Heerwaarden, and M. C. Krol, 2012: A conceptual framework to quantify the influence of convective boundary layer development on carbon dioxide mixing ratios. Atmos. Chem. Phys., 12, 2969-2985, https://doi.org/10.5194/ acp-12-2969-2012.

Prather, M. J., 1996: Time scales in atmospheric chemistry: Theory, GWPS for $\mathrm{CH}_{4}$ and $\mathrm{CO}$, and runaway growth. Geophys. Res. Lett., 23, 2597-2600, https://doi.org/10.1029/ 96GL02371.

Singh, A., and K. Gupta, 1984: Linear wave propagation in nonhomogeneous viscoelastic semi-infinite rod. Indian J. Pure Appl. Math., 15 (2), 171-186.

Stull, R., 1988: An Introduction to Boundary Layer Meteorology. Springer Netherlands, $670 \mathrm{pp}$.

Wang, W., K. Davis, B. D. Cook, P. S. Bakwin, C. Yi, M. P. Butler, and D. M. Ricciuto, 2005: Surface layer $\mathrm{CO}_{2}$ budget and advective contributions to measurements of net ecosystematmosphere exchange of $\mathrm{CO}_{2}$. Agric. For. Meteor., 135, 202-214, https://doi.org/10.1016/j.agrformet.2005.11.018.

Williamson, D. L., J. T. Kiehl, V. Ramanatha, R. E. Dickinson, and J. J. Hack, 1987: Description of NCAR Community Climate Model (CCM1). NCAR Tech. Note NCAR/TN-285+STR, 112 pp., https://doi.org/10.5065/D6TB14WH.

Wyngaard, J., and R. A. Brost, 1984: Top-down and bottom-up diffusion of a scalar in the convective boundary layer. J. Atmos. Sci., 41, 102-112, https://doi.org/10.1175/1520-0469(1984)041<0102: TDABUD $>2.0 . \mathrm{CO} ; 2$.

Yi, C., K. J. Davis, P. S. Bakwin, B. W. Berger, and L. C. Marr, 2000: Influence of advection on measurements of the net ecosystem atmosphere exchange of $\mathrm{CO}_{2}$ from a very tall tower. J. Geophys. Res., 105, 9991-9999, https://doi.org/ 10.1029/2000JD900080.

Zoppou, C., and J. Knight, 1999: Analytical solution of a spatially variable coefficient advection-diffusion equation in up to three dimensions. Appl. Math. Model., 23, 667-685, https://doi.org/ 10.1016/S0307-904X(99)00005-0. 